\title{
Controlling the Isolation and Pairing of Aluminum in Chabazite Zeolites Using Mixtures of Organic and Inorganic Structure-Directing Agents
}

\author{
John R. Di Iorio, Rajamani Gounder* \\ SUPPORTING INFORMATION
}

\section{Section S.1. Synthesis of SSZ-13(5) Zeolites}

The synthesis recipe for SSZ-13(5) was adapted from the procedure described by Fickel et al. ${ }^{1}$, which is a modified version of the original synthesis reported by Zones ${ }^{2}$. A synthesis molar ratio of $1 \mathrm{SiO}_{2} / 0.031 \mathrm{Al}_{2} \mathrm{O}_{3} / 0.017 \mathrm{TMAdaOH} / 0.770 \mathrm{Na}_{2} \mathrm{O} / 12.1 \mathrm{H}_{2} \mathrm{O}$ was used. A typical synthesis involved adding $3.14 \mathrm{~g}$ of a $1 \mathrm{M} \mathrm{NaOH}$ solution (3.3 wt $\% \mathrm{NaOH}$, Alfa Aesar) to $4.82 \mathrm{~g}$ of deionized water (18.2 M $\Omega$ ) in a PFA jar and stirring the solution for 15 minutes at ambient conditions. $24.10 \mathrm{~g}$ of sodium silicate (10.6 wt $\% \mathrm{Na}_{2} \mathrm{O}, 25.6 \mathrm{wt} \% \mathrm{SiO}_{2}$; Sigma Aldrich) was added and homogenized for 15 minutes under ambient conditions. $0.37 \mathrm{~g}$ of $\mathrm{NH}_{4}-\mathrm{Y}$ zeolite (Zeolyst $\mathrm{CBV} 300, \mathrm{Si} / \mathrm{Al}=2.6$ ) were added and the mixture was homogenized for 30 minutes under ambient conditions. $1.58 \mathrm{~g}$ of a $1 \mathrm{M}$ TMAdaOH solution ( $25 \mathrm{wt} \%$ Sachem) was then added to the mixture and stirred for 30 minutes under ambient conditions. All synthesis reagents were used without further purification. The synthesis solution was then transferred to a $45 \mathrm{ml}$ Teflon-lined stainless steel autoclave (Parr Instruments) and placed in a forced convection oven (Yamato DKN-402C) at $413 \mathrm{~K}$ and rotated at $60 \mathrm{RPM}$ for 6 days. 


\section{Section S.2. Synthesis of SSZ-13(15, 1) and SSZ-13(25, 1) Zeolites}

The synthesis recipe for $\operatorname{SSZ}-13(15,1)$ and $\operatorname{SSZ}(25,1)$ zeolites was adapted from the procedure described by Deka et al. $^{3}$, which is a modified version of the original synthesis reported by Zones ${ }^{4}$. A synthesis molar ratio of $1 \mathrm{SiO}_{2} / \mathrm{X} \mathrm{Al}_{2} \mathrm{O}_{3} / 0.25 \mathrm{TMAdaOH} / 0.25 \mathrm{Na}_{2} \mathrm{O} / 44 \mathrm{H}_{2} \mathrm{O}$ was used, where $\mathrm{X}=0.133$ or 0.080 for $\mathrm{Si} / \mathrm{Al}=15$ and 25 , respectively. A typical synthesis involved adding $8.865 \mathrm{~g}$ of a $1 \mathrm{M}$ aqueous TMAdaOH solution (25 wt $\%$, Sachem) to $8.090 \mathrm{~g}$ of deionized $\mathrm{H}_{2} \mathrm{O}(18.2 \mathrm{M} \Omega$ ) to a perfluoroalkoxy alkane (PFA) jar and stirring the solution under ambient conditions for 15 minutes. Next, 0.109 or $0.065 \mathrm{~g}$ (for $\mathrm{Si} / \mathrm{Al}=15$ or 25$)$ of $\mathrm{Al}(\mathrm{OH})_{3}(98 \mathrm{wt} \%$, SPI Pharma) and $1.284 \mathrm{~g}$ of a $5 \mathrm{M}$ sodium hydroxide solution $(\mathrm{NaOH}: 16.7 \mathrm{wt} \% \mathrm{NaOH}$ in deionized water; $\mathrm{NaOH}$ pellets $98 \mathrm{wt} \%$, Alfa Aesar) was added to the aqueous TMAdaOH solution and the mixture was stirred under ambient conditions for 15 minutes to homogenize the contents. Finally, $3.150 \mathrm{~g}$ of colloidal silica (Ludox HS40, $40 \mathrm{wt} \%$, Sigma Aldrich) was added to the mixture and stirred for $2 \mathrm{~h}$ under ambient conditions. All synthesis reagents were used without further purification. The synthesis solution was then transferred to a $45 \mathrm{ml}$ Teflon-lined stainless steel autoclave (Parr Instruments) and placed in a forced convection oven (Yamato DKN-402C) at 433 $\mathrm{K}$ and rotated at $40 \mathrm{RPM}$ for 6 days. 


\section{Section S.3. Synthesis of Pure-silica Chabazite (Si-CHA).}

Pure $\mathrm{SiO}_{2}$ chabazite was synthesized following the procedure reported by Díaz-Cabañas et al. ${ }^{5}$ using a synthesis solution molar ratio of $1 \mathrm{SiO}_{2} / 0.5 \mathrm{TMAdaOH} / 0.5 \mathrm{HF} / 3 \mathrm{H}_{2} \mathrm{O}$. In a typical synthesis $13 \mathrm{~g}$ of tetraethylorthosilicate (TEOS; $98 \mathrm{wt} \%$, Sigma Aldrich) were added to a PFA jar containing $25.849 \mathrm{~g}$ of a $1 \mathrm{M}$ TMAdaOH solution ( $25 \mathrm{wt} \%$, Sachem) and stirred under ambient conditions. Ethanol, formed from the hydrolysis of TEOS, and excess water were then evaporated to reach the target molar ratios by placing the TEOS and TMAdaOH solution under flowing air while stirring at ambient conditions. During the evaporation process, once the solution mass came within $5 \mathrm{~g}$ of the desired final mass, an additional $10 \mathrm{~g}$ of water were added to ensure complete hydrolysis of TEOS and to allow additional time to completely evaporate the ethanol. This rehydration process was performed twice during the evaporation step. As the synthesis solution neared the desired $\mathrm{H}_{2} \mathrm{O} / \mathrm{SiO}_{2}$ ratio of 3 , the solution began to solidify and had to be stirred by hand for three additional hours in order to complete the water evaporation process. Once the synthesis solution had reached the desired $\mathrm{H}_{2} \mathrm{O} / \mathrm{SiO}_{2}$ ratio, $1.275 \mathrm{~g}$ of concentrated hydrofluoric acid (HF; $48 \mathrm{wt} \%$, Sigma Aldrich) was added dropwise to the synthesis and homogenized for 15 minutes. Caution: when working with hydrofluoric acid use appropriate personal protective equipment, ventilation, and other safety measures. Upon addition of HF to the solidified synthesis solution, the solution immediately became a thick paste that became more solution-like under stirring. The solution was then left to sit uncovered under ambient conditions for 30 minutes to allow for any residual $\mathrm{HF}$ to evaporate before transferring the solution to a $45 \mathrm{ml}$ Teflon-lined stainless steel autoclave (Parr Instruments) and heated in a forced convection oven (Yamato DKN-402C) at 423 $\mathrm{K}$ under rotation at $40 \mathrm{RPM}$ for $40 \mathrm{~h}$. All synthesis reagents were used without further purification. 


\section{Section S.4. Synthesis and Characterization of SSZ-13 Zeolites without $\mathrm{Na}^{+}$}

For the synthesis of $\mathrm{Na}^{+}$-free SSZ-13 zeolites in hydroxide media, a molar ratio of $1 \mathrm{SiO}_{2} /$ $\mathrm{X} \mathrm{Al}_{2} \mathrm{O}_{3} / 0.5 \mathrm{TMAdaOH} / 44 \mathrm{H}_{2} \mathrm{O}$ was used, where $\mathrm{X}=0.20,0.133,0.10,0.08,0.067$, or 0.033 for $\mathrm{Si} / \mathrm{Al}=10,15,20,25,30$ and 60 , respectively. In a typical synthesis $14.07 \mathrm{~g}$ of a 1M TMAdaOH solution (25 wt\% Sachem) were added to $12.841 \mathrm{~g}$ of deionized water $(18.2 \mathrm{M} \Omega)$ in a PFA jar and stirred for 15 minutes under ambient conditions. Next $0.260,0.173,0.130,0.104,0.087$, or 0.043 $\mathrm{g}\left(\mathrm{Si} / \mathrm{Al}=10,15,20,25,30\right.$, and 60 , respectively) of $\mathrm{Al}(\mathrm{OH})_{3}(98 \mathrm{wt} \%$, SPI Pharma) were added to the $\mathrm{TMAda}^{+}$solution and the mixture was homogenized for 15 minutes under ambient conditions. Then $5 \mathrm{~g}$ of colloidal silica (Ludox HS40, $40 \mathrm{wt} \%$, Sigma Aldrich) were added to the mixture and the contents were stirred for $2 \mathrm{~h}$ at ambient conditions until a homogeneous solution was obtained. All synthesis reagents were used without further purification. The synthesis solution was then transferred to a $45 \mathrm{ml}$ Teflon-lined stainless steel autoclave (Parr Instruments) and heated in a forced convection oven (Yamato DKN-402C) at $433 \mathrm{~K}$ and rotated at $40 \mathrm{RPM}$ for 6 days. 


\section{Section S.5. Synthesis of SSZ-13 Zeolites at Constant $\mathrm{Na}^{+} / \mathrm{TMAda}^{+}$and Varied Si/Al Ratio}

SSZ-13 zeolites were synthesized at a constant $\mathrm{Na}^{+} / \mathrm{TMAda}^{+}$ratio of $2 / 3$ and with gel $\mathrm{Si} / \mathrm{Al}$ ratios ranging from 10-200 in hydroxide media. A synthesis gel molar ratio of $1 \mathrm{SiO}_{2} / \mathrm{X} \mathrm{Al}_{2} \mathrm{O}_{3} /$ $0.3 \mathrm{TMAdaOH} / 0.2 \mathrm{NaOH} / 44 \mathrm{H}_{2} \mathrm{O}$ was used, where $\mathrm{X}=0.40,0.20,0.13,0.08,0.04,0.02$, and 0.01 , for $\mathrm{Si} / \mathrm{Al}$ ratios of $5,10,15,25,50,100$, and 200 , respectively. In a typical synthesis 8.443 $\mathrm{g}$ of a 1M TMAdaOH solution ( $25 \mathrm{wt} \%$ Sachem) were added to $15.698 \mathrm{~g}$ of deionized water (18.2 $\mathrm{M} \Omega$ ) in a PFA jar and stirred for 15 minutes under ambient conditions. Next 0.519, 0.260, 0.173, $0.104,0.052,0.026$, or $0.013 \mathrm{~g}\left(\mathrm{Si} / \mathrm{Al}=5,10,15,25,50,100\right.$, and 200 , respectively) of $\mathrm{Al}(\mathrm{OH})_{3}$ (98 wt \%, SPI Pharma) were added to the TMAda ${ }^{+}$solution and the mixture was homogenized for 15 minutes under ambient conditions. Then $5 \mathrm{~g}$ of colloidal silica (Ludox HS40, $40 \mathrm{wt} \%$, Sigma Aldrich) were added to the mixture and the contents were stirred for $2 \mathrm{~h}$ at ambient conditions. All synthesis reagents were used without further purification. The synthesis gel was then transferred to a $45 \mathrm{ml}$ Teflon-lined stainless steel autoclave (Parr Instruments) and heated in a forced convection oven (Yamato DKN-402C) at $433 \mathrm{~K}$ and rotated at $40 \mathrm{RPM}$ for 6 days. 
Section S.6. Synthesis of SSZ-13 Zeolites at $\mathrm{Si} / \mathrm{Al}=15$ and 25 at Constant $\left(\mathrm{Na}^{+}+\mathrm{TMAda}^{+}\right) / \mathrm{Al}$ with Varying $\mathrm{Na}^{+} / \mathrm{TMAda}^{+}$Ratio

The synthesis of SSZ-13 zeolites in synthesis solutions with varying charge density, but with constant total charge, was performed at a $\mathrm{Si} / \mathrm{Al}=15$ and 25 at a constant ratio of $\left(\mathrm{Na}^{+}+\mathrm{TMAda}^{+}\right) / \mathrm{Al}=7.5$ while letting the $\mathrm{Na}^{+} / \mathrm{TMAda}^{+}$ratio vary from 0 to 3 . A synthesis solution molar ratio of $1 \mathrm{SiO}_{2} / \mathrm{Y} \mathrm{Al}_{2} \mathrm{O}_{3} / \mathrm{X}$ TMAdaOH$/(0.5-\mathrm{X}) \mathrm{NaOH} / 44 \mathrm{H}_{2} \mathrm{O}$ was used, where $\mathrm{X}=$ $0.50,0.40,0.33,0.30,0.25,0.20,0.17,0.14$, and 0.125, for $\mathrm{Na}^{+} / \mathrm{TMAda}^{+}$ratios of $0,1 / 4,1 / 2,2 / 3$, $1,3 / 2,2,2.5$, and 3, respectively, and $\mathrm{Y}=0.133$ and 0.08 for $\mathrm{Si} / \mathrm{Al}=15$ and 25, respectively. In a typical synthesis $14.071,11.257,9.381,8.443,7.036,5.628,4.690,4.020$, or $3.518 \mathrm{~g}$ $\left(\mathrm{Na}^{+} / \mathrm{TMAda}^{+}=0,1 / 4,1 / 2,2 / 3,1,3 / 2,2,2.5\right.$, and 3 , respectively) of a $1 \mathrm{M}$ TMAdaOH solution (25 wt\% Sachem) were added to $12.841,14.269,15.222,15.698,16.412,17.127,17.603,17.943$, or $18.198 \mathrm{~g}\left(\mathrm{Na}^{+} / \mathrm{TMAda}^{+}=0,1 / 4,1 / 2,2 / 3,1,3 / 2,2,2.5\right.$, and 3 , respectively) of deionized water $(18.2 \mathrm{M} \Omega)$ in a PFA jar and stirred for 15 minutes under ambient conditions. Next 0.173 or 0.104 $\mathrm{g}(\mathrm{Si} / \mathrm{Al}=15$ or 25 , respectively $)$ of $\mathrm{Al}(\mathrm{OH})_{3}(98 \mathrm{wt} \%$, SPI Pharma) were added to the TMAda solution and then $0,0.815,1.359,1.630,2.038,2.446,2.717,2.911$, or $3.057 \mathrm{~g}\left(\mathrm{Na}^{+} / \mathrm{TMAda}^{+}=0\right.$, $1 / 4,1 / 2,2 / 3,1,3 / 2,2,2.5$, and 3 , respectively) of a $5 \mathrm{M}$ sodium hydroxide solution ( $\mathrm{NaOH}: 16.7$ wt $\% \mathrm{NaOH}$ in deionized water; $\mathrm{NaOH} 98$ wt $\%$ Alfa Aesar) were added and the mixture was homogenized for 15 minutes under ambient conditions. Then $5 \mathrm{~g}$ of colloidal silica (Ludox HS40, $40 \mathrm{wt} \%$, Sigma Aldrich) were added to the mixture and the contents were stirred for $2 \mathrm{~h}$ at ambient conditions. All synthesis reagents were used without further purification. The synthesis solution was then transferred to a $45 \mathrm{ml}$ Teflon-lined stainless steel autoclave (Parr Instruments) and heated in a forced convection oven (Yamato DKN-402C) at $433 \mathrm{~K}$ and rotated at $40 \mathrm{RPM}$ for 6 days. 
Section S.7. Synthesis of SSZ-13 Zeolites at $\mathrm{Si} / \mathrm{Al}=15$ with Varying $\left(\mathrm{Na}^{+}+\mathrm{TMAda}^{+}\right) / \mathrm{Al}$ and Varying $\mathrm{Na}^{+} / \mathrm{TMAda}^{+}$

Synthesis of SSZ-13 zeolites with varying total charge and charge density were performed at a solution $\mathrm{Si} / \mathrm{Al}$ ratio of 15 while varying the $\mathrm{Na}^{+} / \mathrm{TMAda}^{+}$(charge density) from 0 to 5.6 and $\left(\mathrm{Na}^{+}+\mathrm{TMAda}^{+}\right) / \mathrm{Al}$ (total charge) from 5.4 to 7.1. A synthesis solution molar ratio of $1 \mathrm{SiO}_{2} / 0.133$ $\mathrm{Al}_{2} \mathrm{O}_{3} / \mathrm{X}$ TMAdaOH$/(0.5-\mathrm{X}) \mathrm{NaOH} / 44 \mathrm{H}_{2} \mathrm{O}$ was used, where $\mathrm{X}=0.50,0.37,0.29,0.26,0.21$, 0.16, 0.11, 0.097, and 0.076, for $\mathrm{Na}^{+} / \mathrm{TMAda}^{+}$ratios of $0,0.35,0.70,0.93,1.38,2.09,3.45,4.16$, and 5.55, respectively. In a typical synthesis $8.443,6.254,4.966,4.374,3.547,2.732,1.897,1.636$,

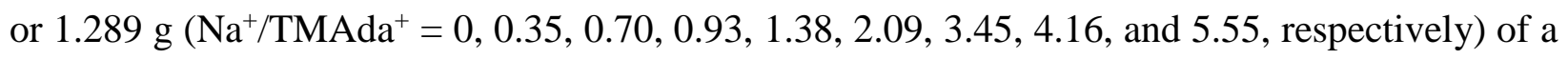
1M TMAdaOH solution (25 wt\% Sachem) were added to 7.704, 8.816, 9.469, 9.770, 10.190, $10.603,11.027,11.160$, or $11.336 \mathrm{~g}\left(\mathrm{Na}^{+} / \mathrm{TMAda}^{+}=0,0.35,0.70,0.93,1.38,2.09,3.45,4.16\right.$, and 5.55 , respectively) of deionized water $(18.2 \mathrm{M} \Omega)$ in a PFA jar and stirred for 15 minutes under ambient conditions. Next $0.104 \mathrm{~g}$ of $\mathrm{Al}(\mathrm{OH})_{3}(98 \mathrm{wt} \%$, SPI Pharma) were added to the TMAda

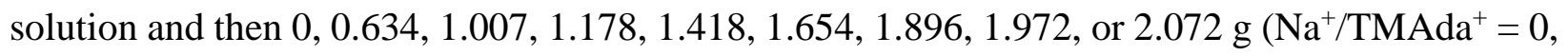
$0.35,0.70,0.93,1.38,2.09,3.45,4.16$, and 5.55 , respectively) of a $5 \mathrm{M}$ sodium hydroxide solution (NaOH: 16.7 wt $\% \mathrm{NaOH}$ in deionized water; $\mathrm{NaOH} 98$ wt\% Alfa Aesar) were added and the mixture was homogenized for 15 minutes under ambient conditions. Then $3 \mathrm{~g}$ of colloidal silica (Ludox HS40, $40 \mathrm{wt} \%$, Sigma Aldrich) were added to the mixture and the contents were stirred for $2 \mathrm{~h}$ at ambient conditions. All synthesis reagents were used without further purification. The synthesis solution was then transferred to a $23 \mathrm{ml}$ Teflon-lined stainless steel autoclave (Parr Instruments) and heated in a forced convection oven (Yamato DKN-402C) at $433 \mathrm{~K}$ and rotated at 40 RPM for 6 days. 
Section S.8. Powder X-Ray Diffraction Patterns of SSZ-13 Zeolites.

Figure S.1. Powder X-ray diffraction patterns for a) SSZ-13(5), b) SSZ-13(15, 1) and, c) SSZ$13(25,1)$.

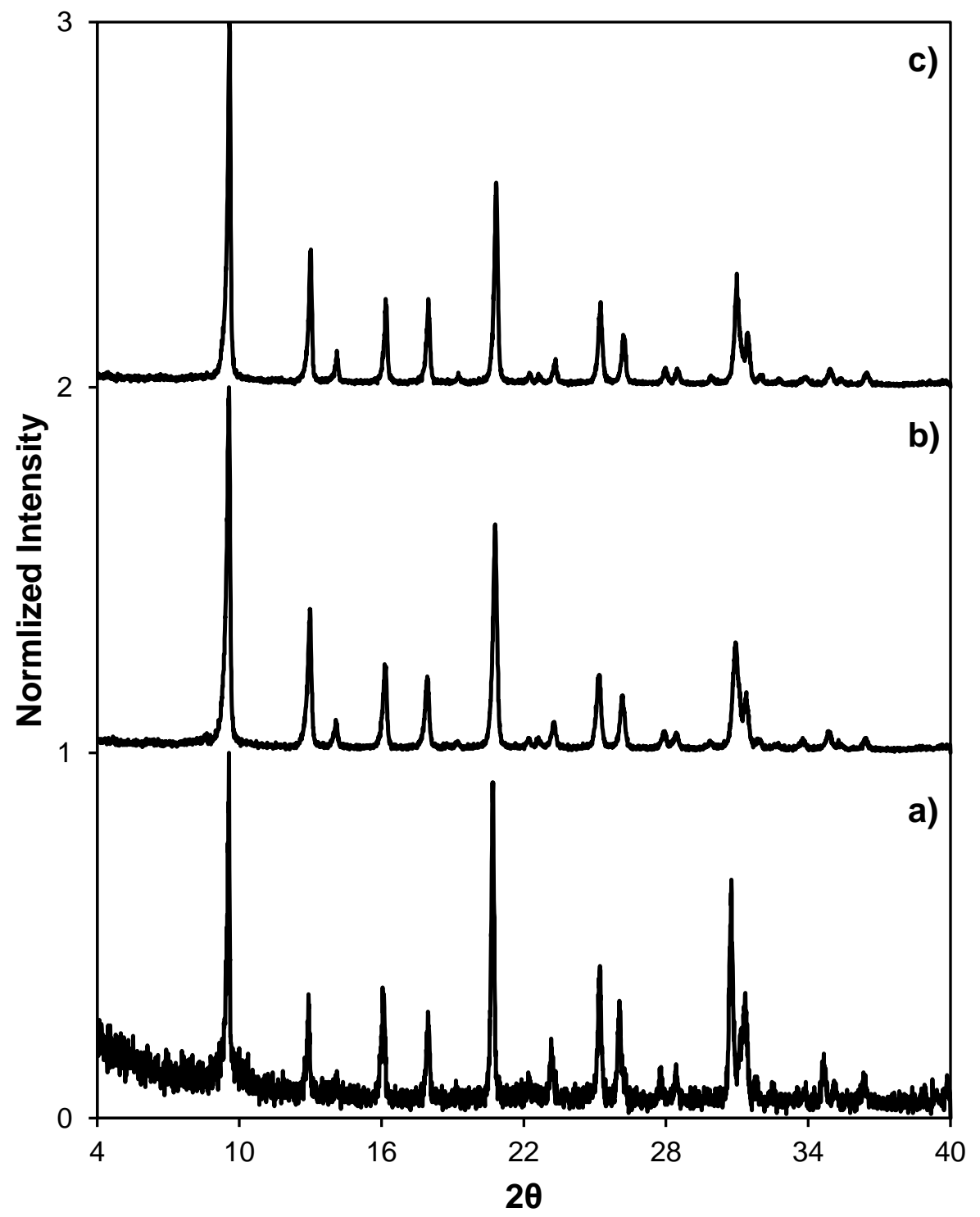


Figure S.2. Powder X-ray diffraction patterns for a) SSZ-13(10, 0), b) SSZ-13(15, 0), c) SSZ13(20, 0), d) SSZ-13(25, 0), e) SSZ-13(30, 0), f) SSZ-13(60, 0), and g) Si-CHA samples synthesized in the absence of $\mathrm{Na}^{+}$at varying $\mathrm{Si} / \mathrm{Al}$ ratios

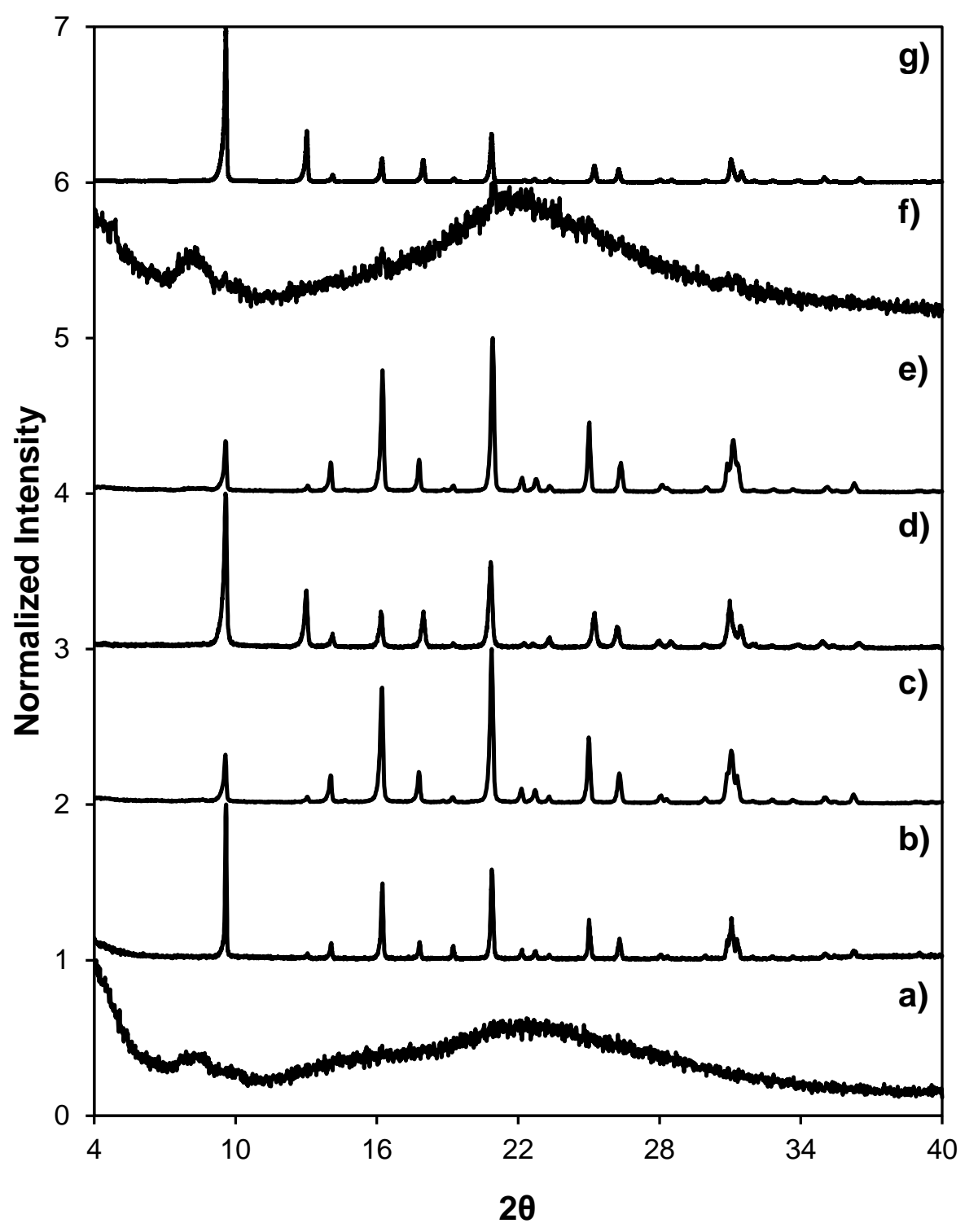


Figure S.3. Powder X-ray diffraction patterns for six independent syntheses of SSZ-13(15, 0)

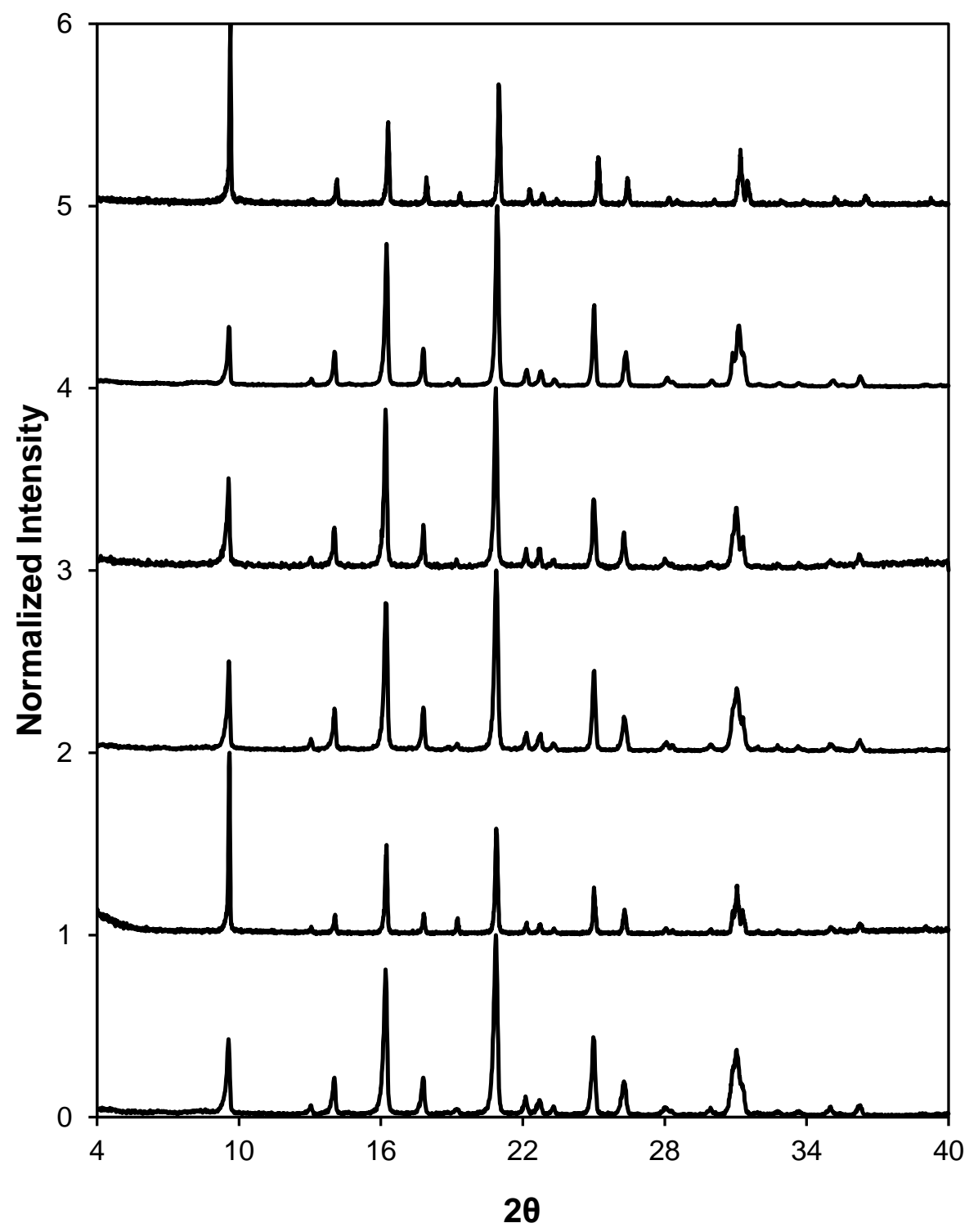


Figure S.4. Powder X-ray diffraction patterns for a) SSZ-13(5, 0.67), b) SSZ-13(10, 0.67), c) SSZ-13(15, 0.67), d) SSZ-13(25, 0.67), e) SSZ-13(50, 0.67), f) SSZ-13(100, 0.67), and g)

SSZ-13(200, 0.67) samples synthesized with constant $\mathrm{Na}^{+} / \mathrm{TMAda}^{+}=0.67$ and varying $\mathrm{Si} / \mathrm{Al}$ ratios

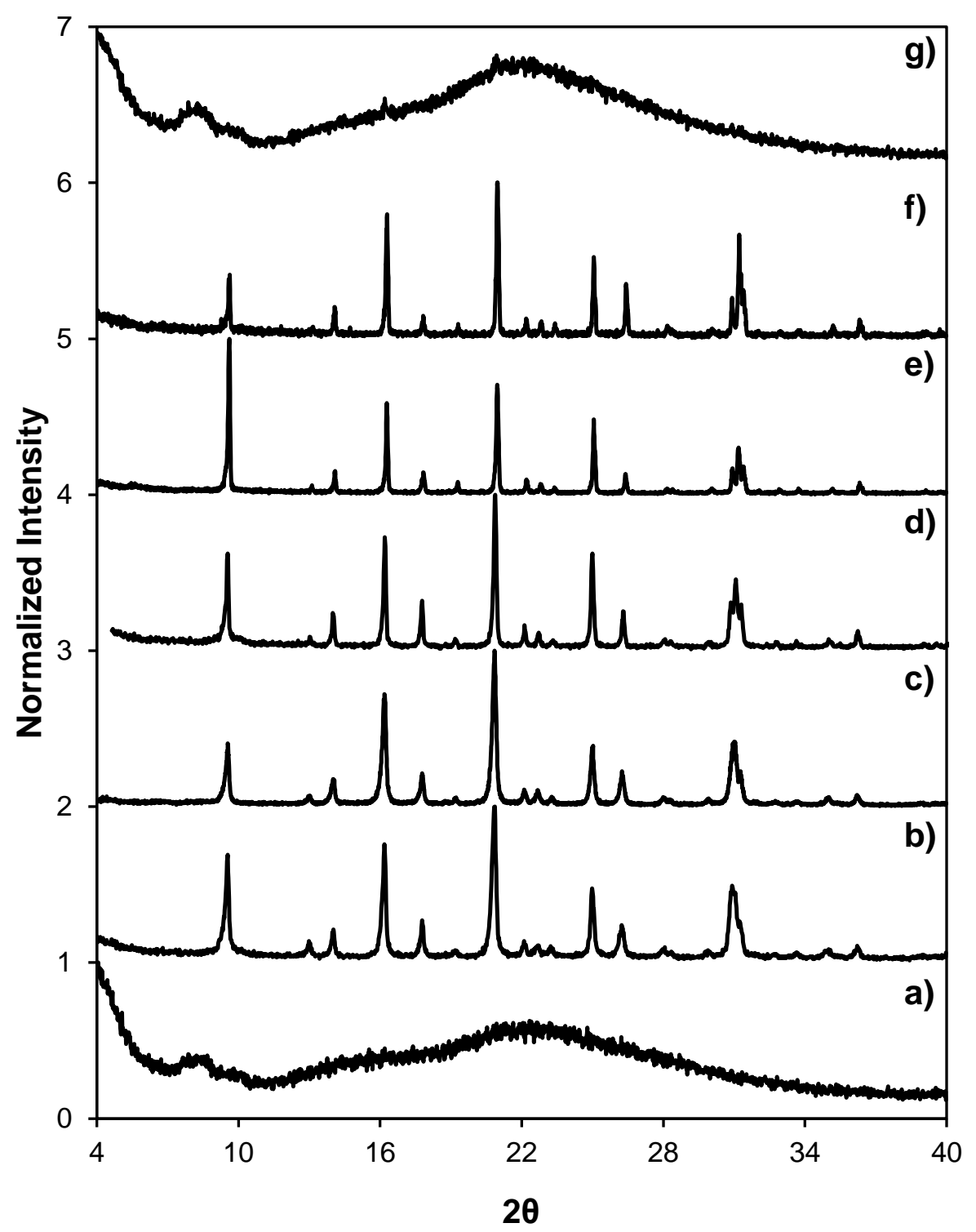


Figure S.5. Powder X-ray diffraction patterns for a) SSZ-13(15, 0), b) SSZ-13(15, 0.25), c) SSZ13(15, 0.50), d) SSZ-13(15, 0.67), e) SSZ-13(15, 1.00), f) SSZ-13(15, 1.50), g) SSZ-13(15, 2.00), h) $\operatorname{MOR}(15,2.50)$, and i) $\operatorname{MOR}(15,3.00)$ samples synthesized with constant solution $\mathrm{Si} / \mathrm{Al}=15$, $\left(\mathrm{Na}^{+}+\mathrm{TMAda}^{+}\right) / \mathrm{Al}$ (total charge), and varying $\mathrm{Na}^{+} / \mathrm{TMAda}^{+}$ratios (charge density)

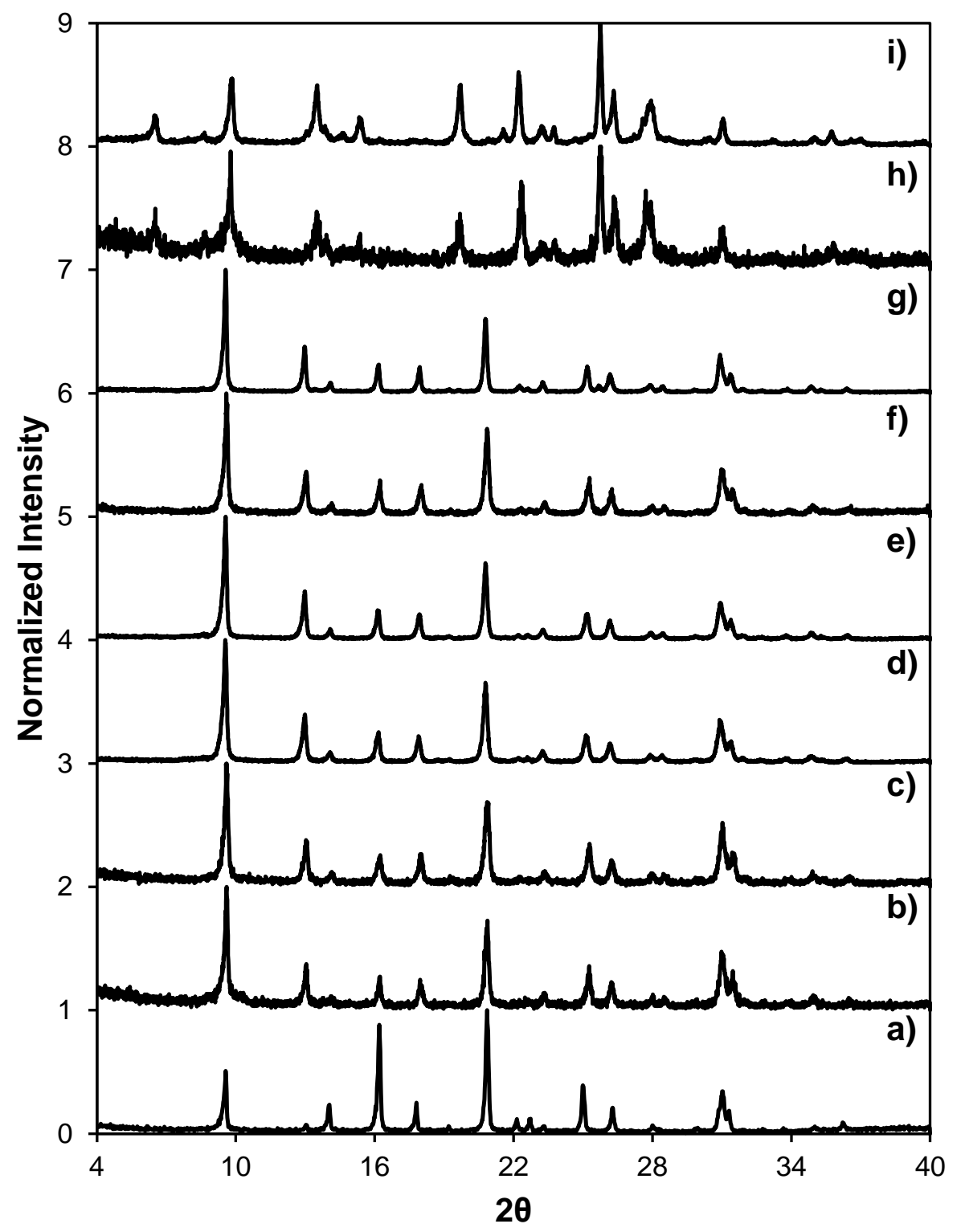


Figure S.6. Powder X-ray diffraction patterns for a) SSZ-13(25, 0), b) SSZ-13(25, 0.50), c) SSZ13(25, 0.67), d) SSZ-13(25, 1.00), and e) SSZ-13(25, 2.00) samples synthesized with constant solution $\mathrm{Si} / \mathrm{Al}=25$ and $\left(\mathrm{Na}^{+}+\mathrm{TMAda}{ }^{+}\right) / \mathrm{Al}$ (total charge), and varying $\mathrm{Na}^{+} / \mathrm{TMAda}^{+}$ratios $($charge density).

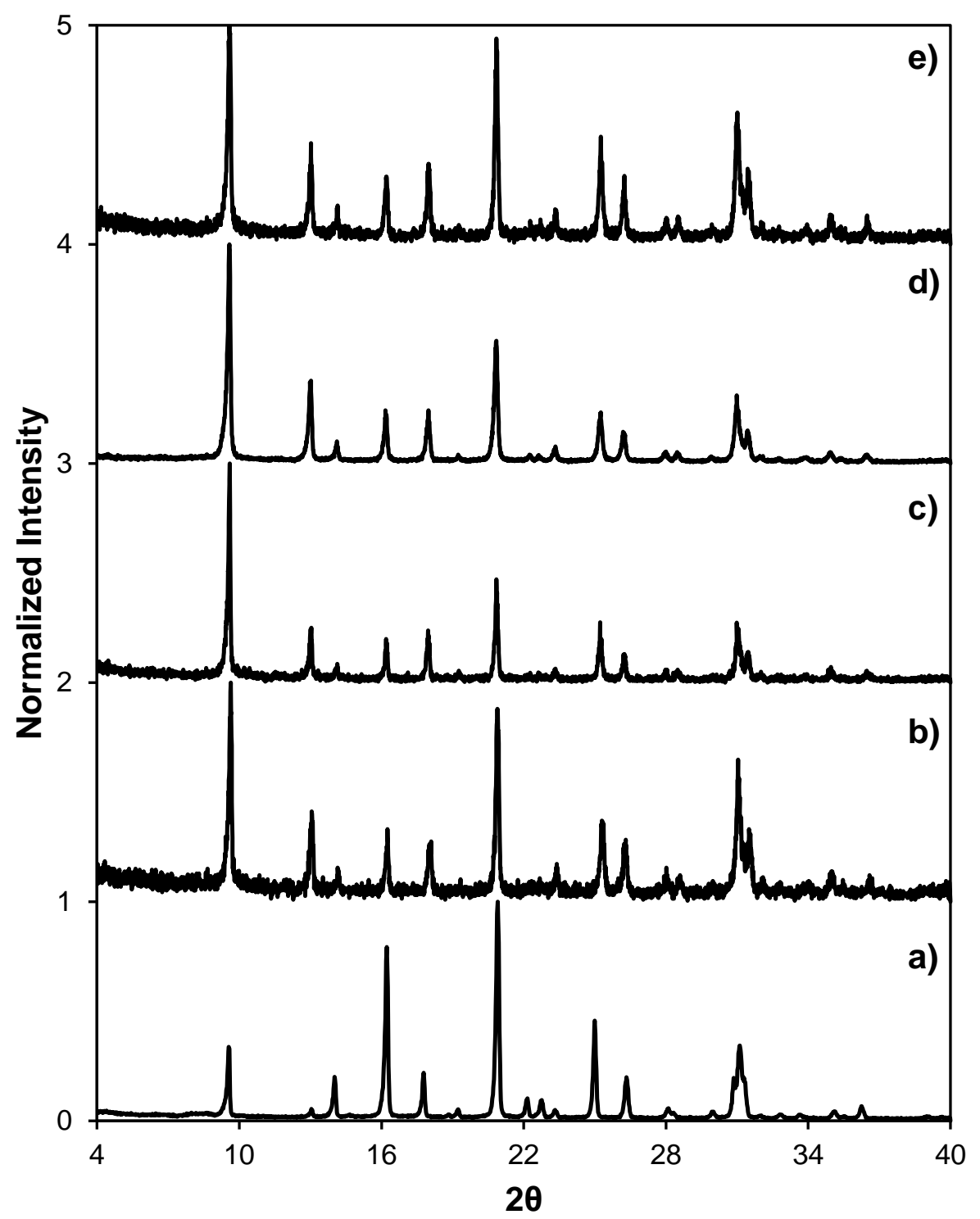


Figure S.7. Powder X-ray diffraction patterns for a) SSZ-13(15, 0), b) SSZ-13(15, 0.35), c) SSZ13(15, 0.70), d) SSZ-13(15, 0.93), e) SSZ-13(15, 1.38), f) SSZ-13(15, 2.09), g) MOR(15, 3.45), h) $\operatorname{MOR}(15,4.16$, and i) $\operatorname{MOR}(15,5.55)$ samples synthesized with constant solution $\mathrm{Si} / \mathrm{Al}=15$ and varying $\left(\mathrm{Na}^{+}+\mathrm{TMAda}{ }^{+}\right) / \mathrm{Al}$ (total charge) and $\mathrm{Na}^{+} / \mathrm{TMAda}^{+}$ratios (charge density).

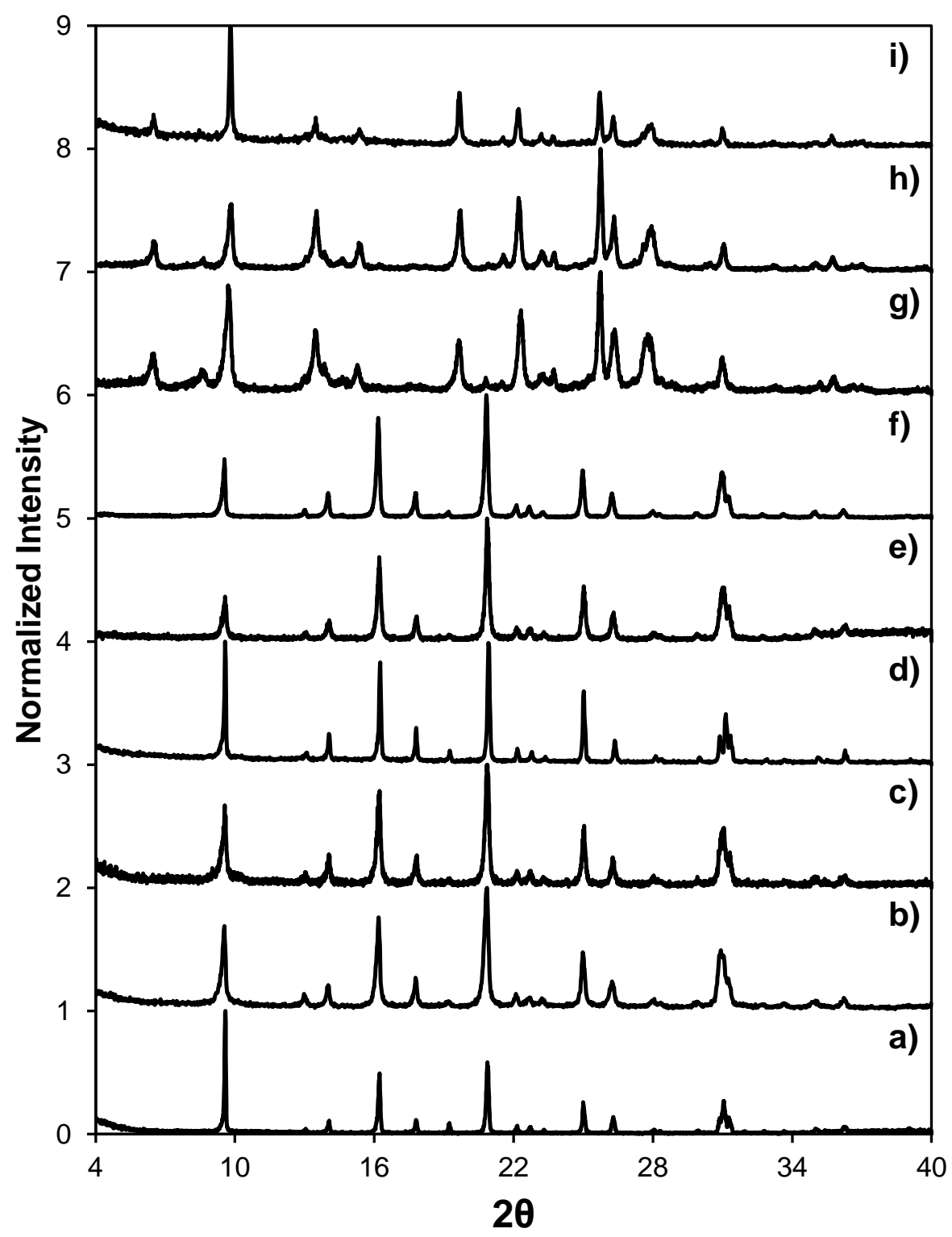


Section S.9. Ar Adsorption Isotherms of Catalyst Samples

Figure S.8. Ar adsorption isotherms at measured at $87 \mathrm{~K}$ on a) SSZ-13(5), b) SSZ-13(15, 1), and c) $\operatorname{SSZ}-13(25,1)$.

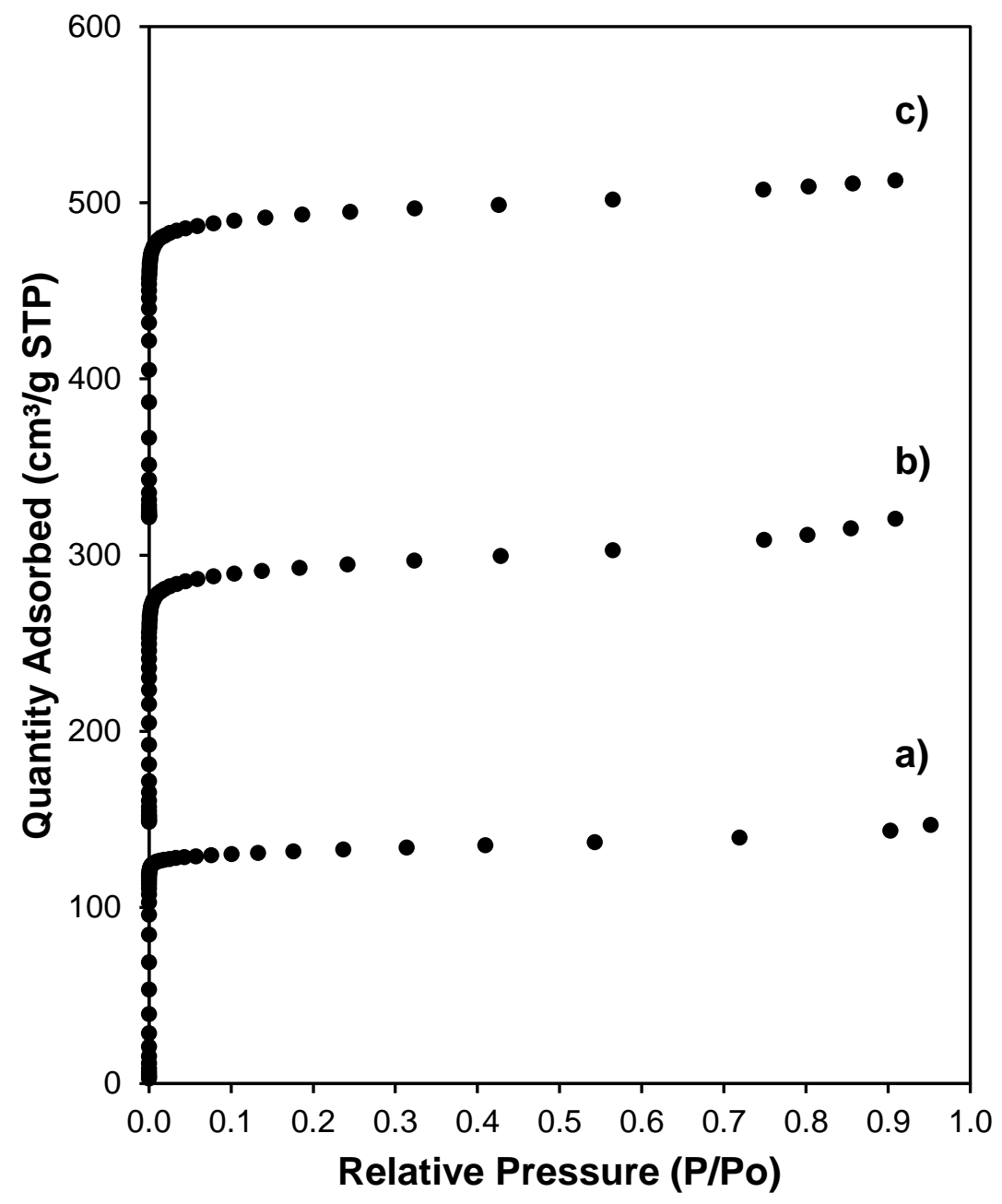


Figure S.9. Ar adsorption isotherms at measured at $87 \mathrm{~K}$ on a) SSZ-13(15, 0), b) SSZ-13(20, 0), c) $\mathrm{SSZ}-13(25,0)$, d) SSZ-13(30, 0), and e) Si-CHA.

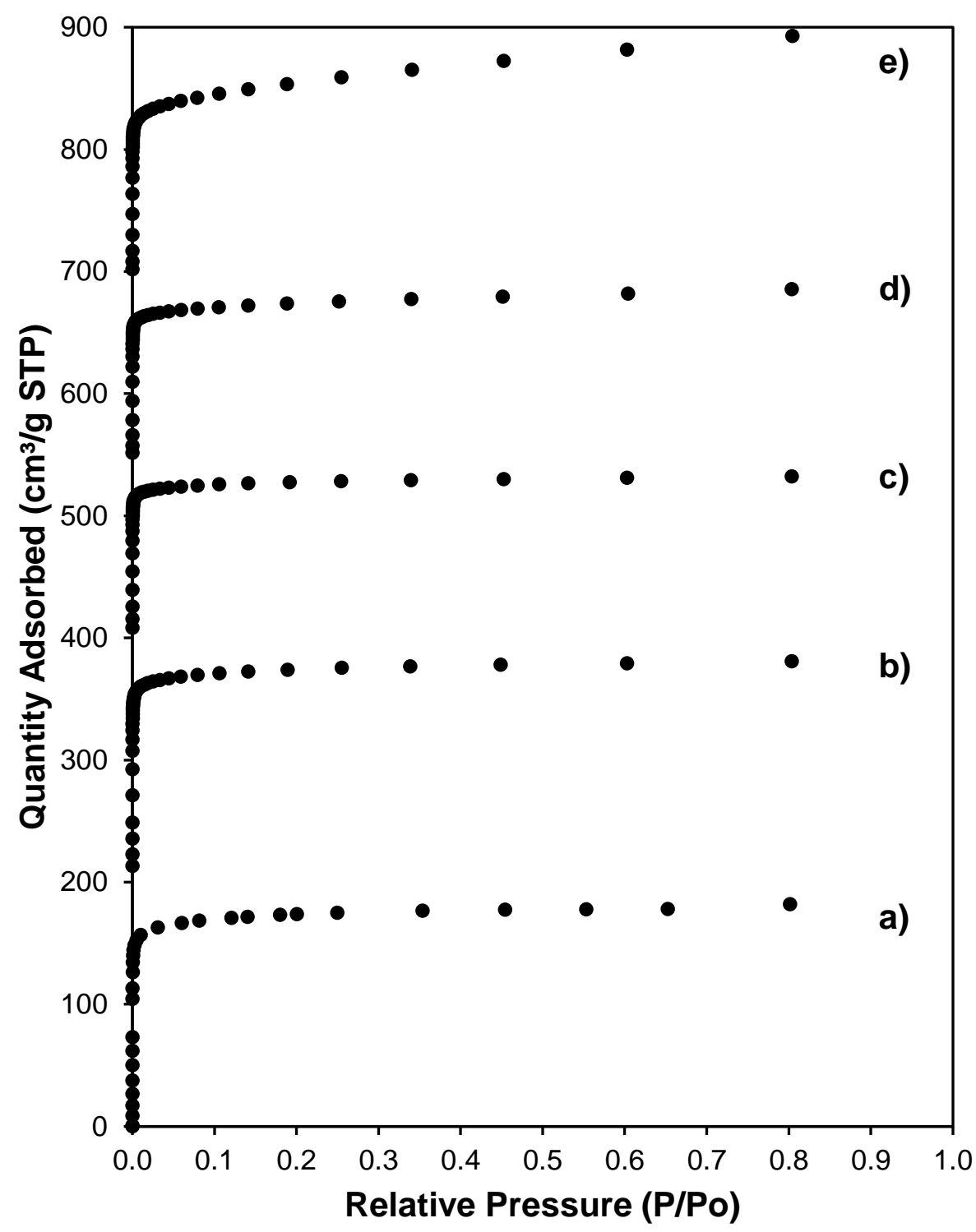


Figure S.10. Ar adsorption isotherms at measured at $87 \mathrm{~K}$ on a) H-SSZ-13(15, 0), b) H-SSZ13(15, 0.25), c) H-SSZ-13(15, 0.50), d) H-SSZ-13(15, 0.67), e) H-SSZ-13(15, 1.00), f) H-SSZ13(15, 1.50), and g) H-SSZ-13(15, 2.00).

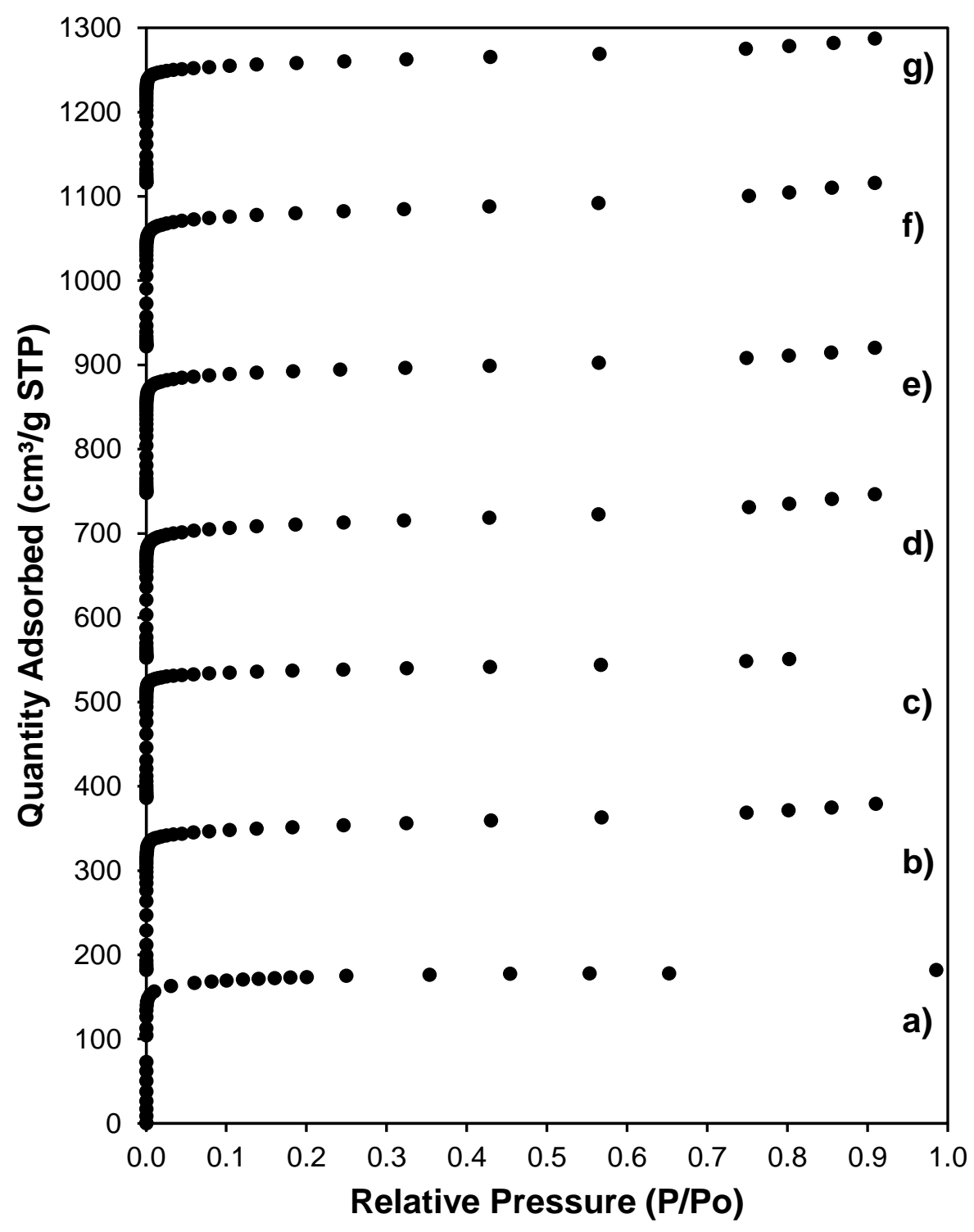


Figure S.11. Ar adsorption isotherms at measured at $87 \mathrm{~K}$ on a) H-SSZ-13(25, 0), b) H-SSZ13(25, 0.25), c) H-SSZ-13(25, 0.50), d) H-SSZ-13(25, 1.00), and e) H-SSZ-13(25, 2.00).

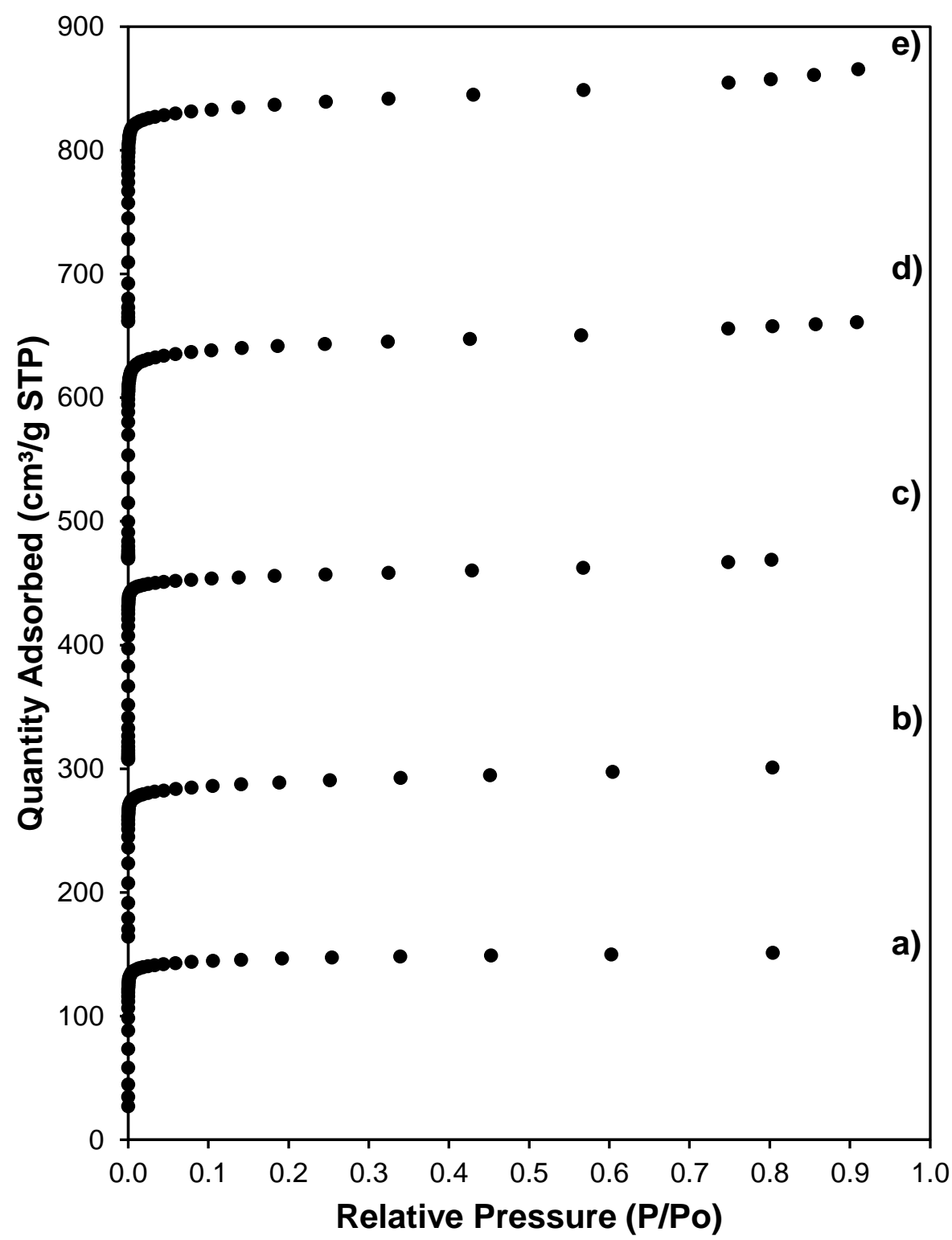


Section S.10. Thermogravimetric Analysis (TGA) to Measure the Organic Content of AsSynthesized Zeolites

Table S.1. Calculation of the number of TMAda ${ }^{+}$per cage for SSZ-13(X,0) and SSZ-13(15,X) with maintaining a constant $\left(\mathrm{Na}^{+}+\mathrm{TMAda}^{+}\right) / \mathrm{Al}$ ratio.

\begin{tabular}{cccc}
\hline Sample ID & Si/Al & TMAda $^{+}$/Al & TMAda $^{+}$Cage \\
\hline SSZ-13(15, 0) & 14.5 & 1.08 & 1.25 \\
SSZ-13(20, 0) & 17.5 & 1.00 & 0.97 \\
SSZ-13(25, 0) & 24.6 & 1.44 & 1.01 \\
SSZ-13(30, 0) & 26.1 & 1.66 & 1.10 \\
\hline SSZ-13(15, 0.25) & 13.5 & 0.93 & 1.15 \\
SSZ-13(15, 0.50) & 14.7 & 0.97 & 1.11 \\
SSZ-13(15, 0.67) & 15.0 & 0.93 & 1.04 \\
SSZ-13(15, 1.00) & 14.8 & 1.04 & 1.18 \\
SSZ-13(15, 1.50) & 15.2 & 1.05 & 1.17 \\
SSZ-13(15, 2.00) & 13.5 & 0.89 & 1.10 \\
\hline
\end{tabular}




\section{Section S.11. ${ }^{27}$ Al Magic Angle Spinning Nuclear Magnetic Resonance (MAS NMR)}

The ${ }^{27}$ Al MAS NMR spectra for SSZ-13 samples synthesized without $\mathrm{Na}^{+}$are shown in Figure S.12 and those synthesized at constant $\mathrm{Si} / \mathrm{Al}=15,\left(\mathrm{Na}^{+}+\mathrm{TMAda}^{+}\right) / \mathrm{Al}$, and varying $\mathrm{Na}^{+} / \mathrm{TMAda}^{+}$are shown in Figure S.13. Tetrahedrally-coordinated Al atoms were characterized by a resonance centered around $60 \mathrm{ppm}$. There was no shoulder present at about $48 \mathrm{ppm}$, representative of pentacoordinated $\mathrm{Al}$, and a minimal (0-5\%) amount of octahedral $\mathrm{Al}$ present at about $0 \mathrm{ppm}^{6-8}$. Spectra were recorded with different sample masses so a quantitative comparison of the line intensities between different samples is not possible because the intensity is a function of the total $\mathrm{Al}$ content in the rotor, therefore the intensity of each H-SSZ-13 spectra was normalized by the maximum intensity in each spectrum to allow for qualitative comparison of the different $\mathrm{H}$ SSZ-13 samples. 
Figure S.12. ${ }^{27} \mathrm{Al}$ MAS NMR spectra for a) H-SSZ-13(15, 0), b) H-SSZ-13(20, 0), c) H-SSZ$13(25,0)$, and d) H-SSZ-13(30, 0)

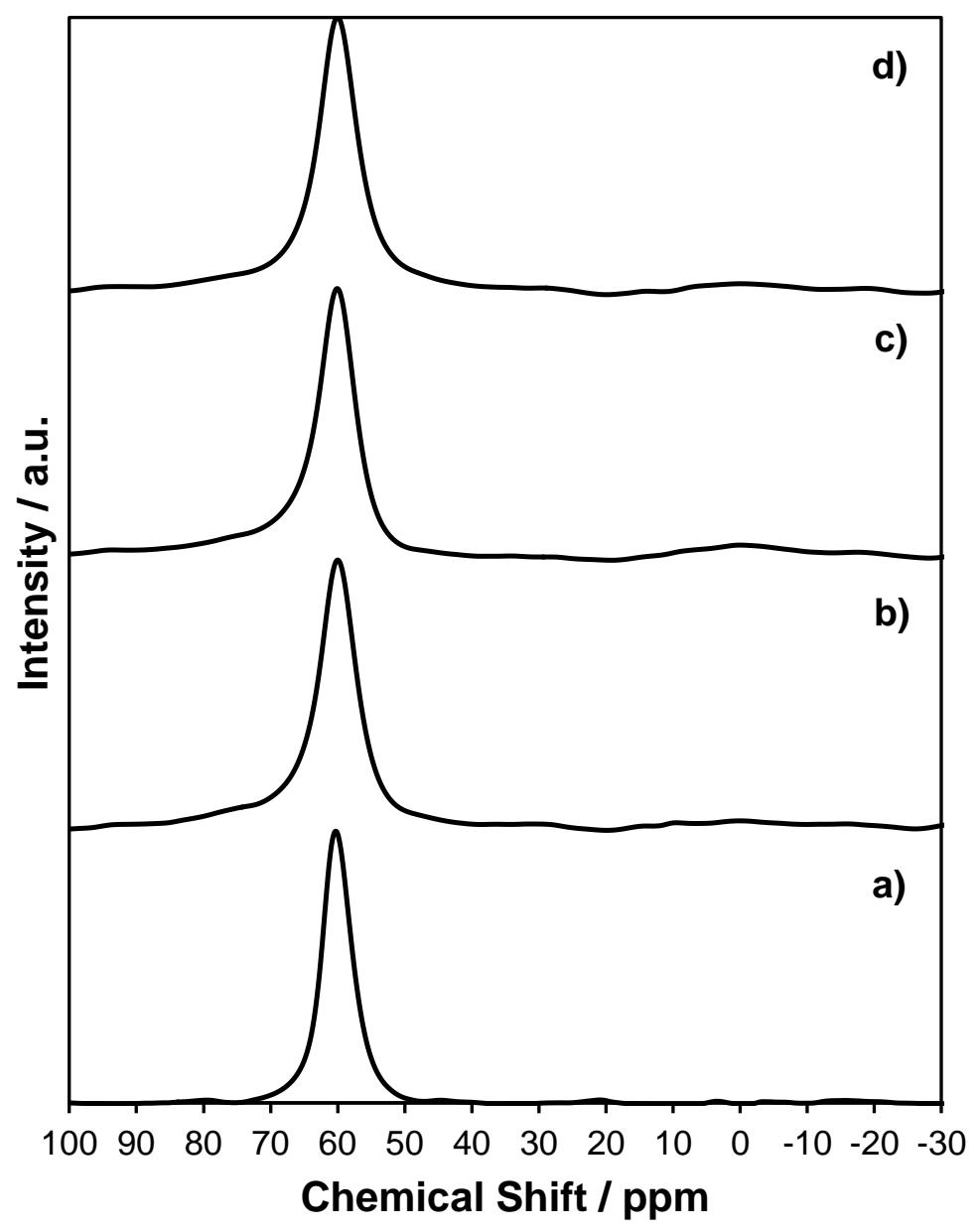


Figure S.13. ${ }^{27} \mathrm{Al}$ MAS NMR spectra for a) H-SSZ-13(15, 0), b) H-SSZ-13(15, 0.25), c) H-SSZ13(15, 0.50), d) H-SSZ-13(15, 0.67), e) H-SSZ-13(15, 1.00), f) H-SSZ-13(15, 1.50), and g) HSSZ-13(15, 2.00)

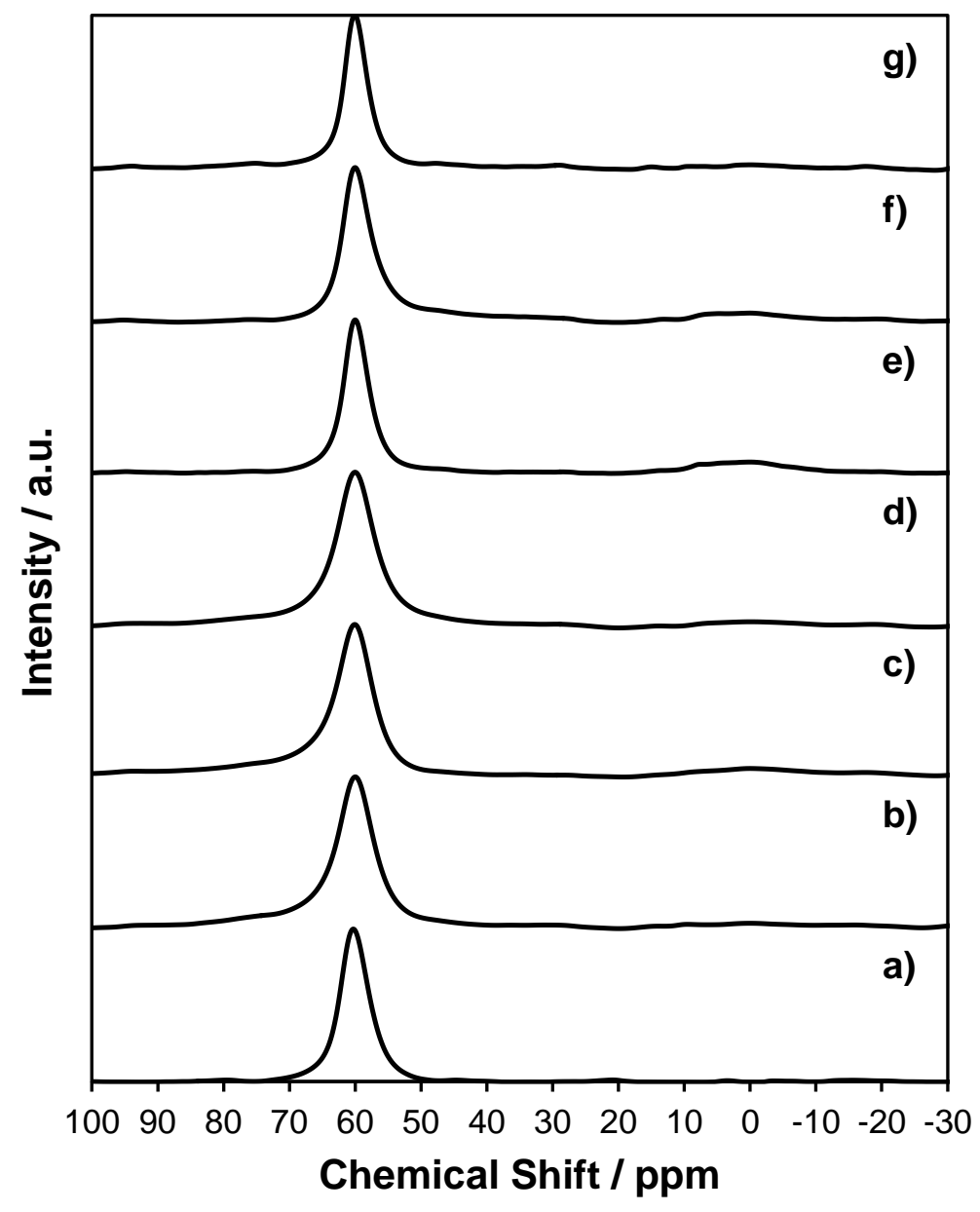




\section{Section S.12. Copper Cation Speciation in SSZ-13 Zeolites.}

Table S.2. $\mathrm{Cu} / \mathrm{Al}$, saturation $\mathrm{Co} / \mathrm{Al}$, predicted $\mathrm{M}^{2+} / \mathrm{Al}$, and residual $\mathrm{H}^{+} / \mathrm{Al}$ on $\mathrm{H}-\mathrm{SSZ}-13(5), \mathrm{H}-$ SSZ-13(15, 1), and H-SSZ-13(25, 1).

\begin{tabular}{|c|c|c|c|c|c|c|c|c|}
\hline Sample & Si/Al & $\begin{array}{c}\text { Micropore } \\
\text { Volume } \\
\left(\mathrm{cm}^{3} \mathrm{~g}_{\mathrm{cat}}{ }^{-1}\right)\end{array}$ & $\mathbf{H}^{+} / \mathbf{A l}^{\mathbf{a}}$ & $\mathrm{Co} / \mathrm{Al}$ & $\mathbf{H}^{+} / \mathbf{A l}^{\mathbf{b}}$ & $\begin{array}{c}\text { Predicted } \\
\mathbf{M}^{2+} / \mathbf{A l}\end{array}$ & $\mathrm{Cu} / \mathrm{Al}$ & $\mathbf{H}^{+} / \mathbf{A l}^{\mathbf{c}}$ \\
\hline \multirow{7}{*}{ SSZ-13(5) } & \multirow{7}{*}{4.5} & \multirow{7}{*}{0.16} & \multirow{7}{*}{0.65} & \multirow{7}{*}{0.19} & \multirow{7}{*}{0.28} & \multirow{7}{*}{0.22} & 0.00 & 0.65 \\
\hline & & & & & & & 0.02 & 0.45 \\
\hline & & & & & & & 0.04 & 0.60 \\
\hline & & & & & & & 0.09 & 0.43 \\
\hline & & & & & & & 0.12 & 0.37 \\
\hline & & & & & & & 0.16 & 0.32 \\
\hline & & & & & & & 0.20 & 0.31 \\
\hline \multirow{8}{*}{ SSZ-13(15, 1) } & \multirow{8}{*}{14.8} & \multirow{8}{*}{0.19} & \multirow{8}{*}{1.02} & \multirow{8}{*}{0.08} & \multirow{8}{*}{0.82} & \multirow{8}{*}{0.09} & 0.00 & 1.02 \\
\hline & & & & & & & 0.03 & 0.94 \\
\hline & & & & & & & 0.09 & 0.81 \\
\hline & & & & & & & 0.12 & 0.79 \\
\hline & & & & & & & 0.21 & 0.68 \\
\hline & & & & & & & 0.37 & 0.58 \\
\hline & & & & & & & 0.41 & 0.55 \\
\hline & & & & & & & 0.44 & 0.51 \\
\hline \multirow{5}{*}{$\operatorname{SSZ}-13(25,1)$} & \multirow{5}{*}{24.1} & \multirow{5}{*}{0.18} & \multirow{5}{*}{0.98} & \multirow{5}{*}{0.04} & \multirow{5}{*}{0.88} & \multirow{5}{*}{0.05} & 0.00 & 0.96 \\
\hline & & & & & & & 0.06 & 0.86 \\
\hline & & & & & & & 0.21 & 0.74 \\
\hline & & & & & & & 0.37 & 0.58 \\
\hline & & & & & & & 0.41 & 0.54 \\
\hline
\end{tabular}

${ }^{\mathrm{a}} \mathrm{H}^{+} / \mathrm{Al}$ measured on the parent $\mathrm{H}-\mathrm{SSZ}-13$ sample

${ }^{\mathrm{b}} \mathrm{H}^{+} / \mathrm{Al}$ measured after saturation with $\mathrm{Co}^{2+}$

${ }^{c} \mathrm{H}^{+} / \mathrm{Al}$ measured after ion-exchange with $\mathrm{Cu}^{2+}$ 
Figure S.14. Number of residual $\mathrm{H}^{+}$sites, normalized to the parent $\mathrm{H}^{+} / \mathrm{Al}$ for each sample, after $\mathrm{Cu}^{2+}$ exchange (circles) as a function of the $\mathrm{Cu} / \mathrm{Al}$ ratio, after $\mathrm{Co}^{2+}$ saturation (squares) on $\mathrm{H}-\mathrm{SSZ}$ 13(5) (black), H-SSZ-13(15, 1) (dark grey), and H-SSZ-13(25, 1) (grey). Dashed lines indicate predictions from statistical calculations of random $\mathrm{Al}$ distributions and the sequential exchange of $\mathrm{Cu}^{2+}$ at paired $\mathrm{Al}$ sites (2 $\mathrm{Al}$ in 6-MR) followed by exchange of $[\mathrm{CuOH}]^{+}$at isolated $\mathrm{Al}$ sites.

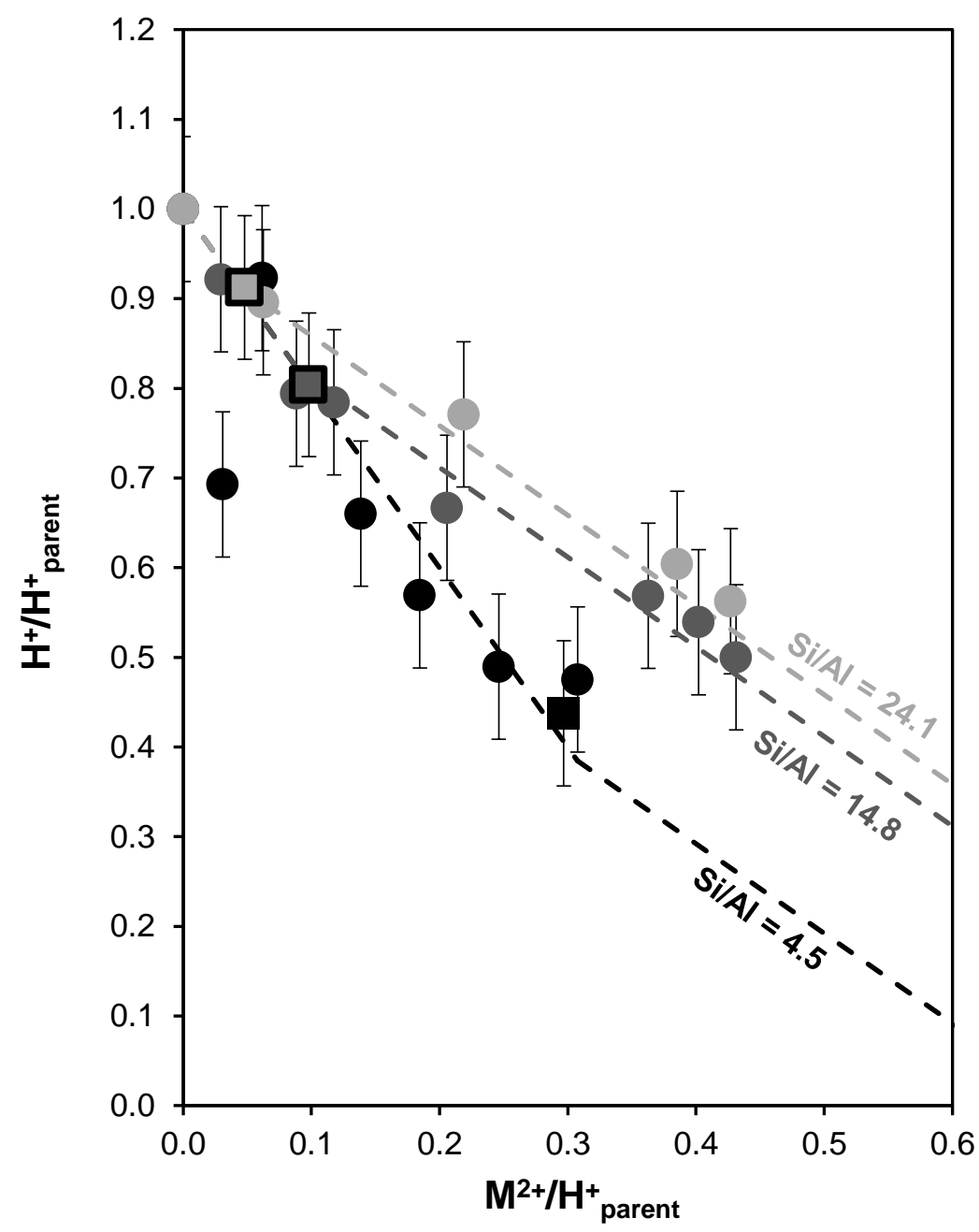




\section{Section S.13. Statistical Estimates of Paired Aluminum in CHA Zeolites.}

Figure S.15: Statistical calculations for the random distribution of $\mathrm{Al}$ in $\mathrm{CHA}$ at varying $\mathrm{Si} / \mathrm{Al}$ ratios, obeying Lowenstein's Rule ${ }^{9}$. The circles denote $\mathrm{SSZ}-13$ materials synthesized at $\mathrm{Si} / \mathrm{Al}=5$, 15 , and 25 and correspond to theoretical $\mathrm{M}^{2+} / \mathrm{Al}=0.22,0.09$, and 0.05 , respectively.

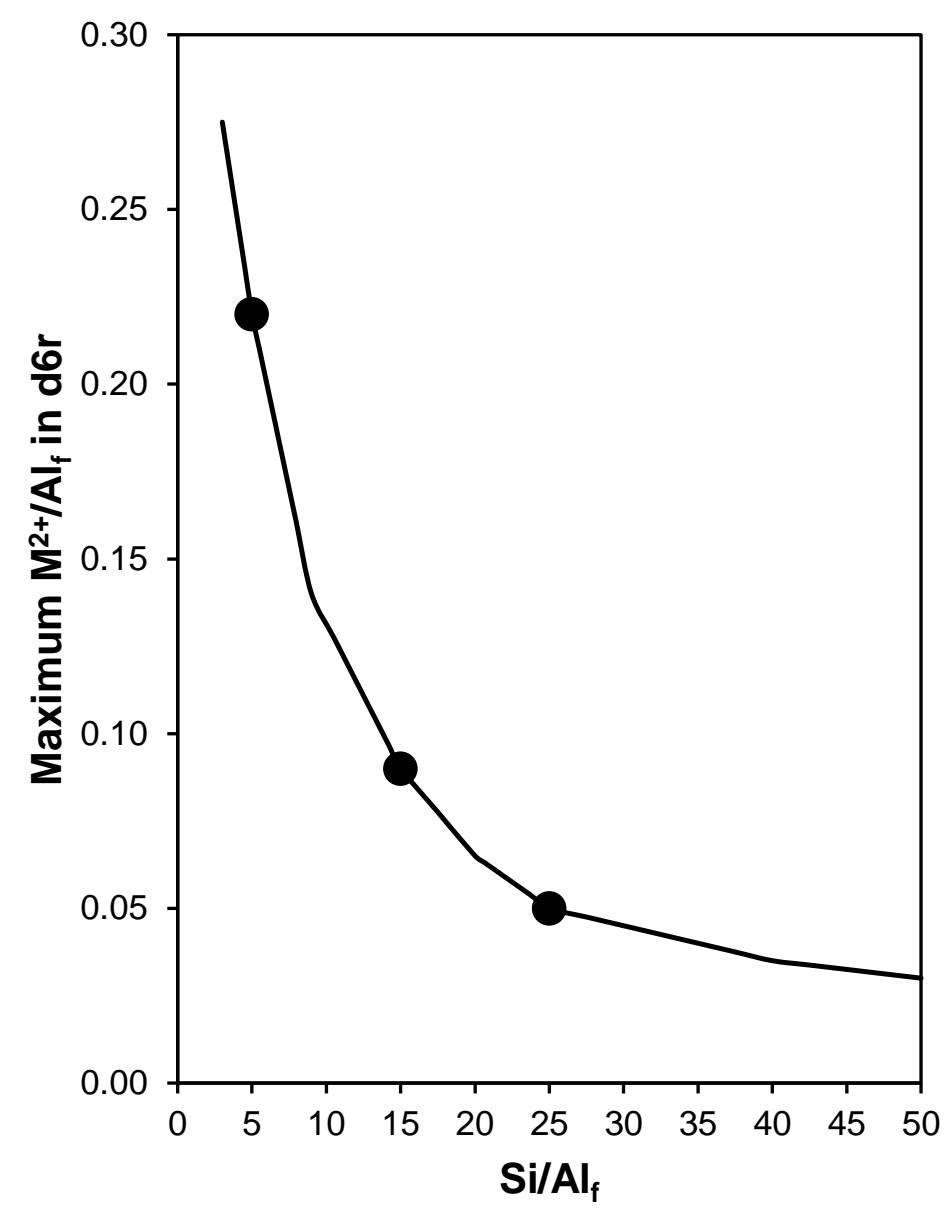




\section{Section S.14. Derivation of Cobalt Ion-Exchange Isotherm}

In order to demonstrate complete saturation of H-SSZ-13 samples with $\mathrm{Co}^{2+}$, an ionexchange isotherm was measured on both H-SSZ-13(15, 0) and H-SSZ-13 $(15,1)$ using $\mathrm{Co}\left(\mathrm{NO}_{3}\right)_{2}$ molarities ranging from $0.005 \mathrm{M}-0.5 \mathrm{M}$ and the procedure described previously in the main text. The Langmuirian ion-exchange isotherm was derived by first assuming that the total $\mathrm{Al}$ atoms on a given sample $\left(\mathrm{Al}_{\text {tot }}\right)$ are equal to the number of $\mathrm{Al}$ atoms that cannot exchange a cation $\left(\mathrm{Al}_{\text {non,exch }}\right)$ and the number of $\mathrm{Al}$ atoms that can be ion-exchanged ( $\left.\mathrm{Al}_{\text {exch }}\right)$ :

$$
A l_{\text {tot }}=A l_{\text {non,exch }}+A l_{\text {exch }}
$$

The number of $\mathrm{Al}_{\text {exch }}$ atoms is equal to the sum of the number of isolated $\mathrm{Al}$ sites, which can exchange a monovalent cation $\left(A l_{\text {iso }}^{\text {tot }}\right)$, and twice the number of paired Al sites, which can exchange a divalent cation $\left(A l_{\text {pair }}^{\text {tot }}\right)$ :

$$
A l_{\text {exch }}=A l_{\text {iso }}^{\text {tot }}+2 A l_{\text {pair }}^{\text {tot }}
$$

A mole balance on each of the $A l_{\text {iso }}^{\text {tot }}$ and $A l_{\text {pair }}^{\text {tot }}$ terms describes the types of cations they can exchange:

$$
\begin{aligned}
& A l_{\text {iso }}^{\text {tot }}=A l_{\text {iso }}^{H^{+}} \\
& A l_{\text {pair }}^{\text {tot }}=A l_{\text {pair }}^{H^{+}, H^{+}}+A l_{\text {pair }}^{\text {Co }}{ }^{2+}
\end{aligned}
$$

where $\mathrm{Al}_{\text {iso }}^{\mathrm{H}^{+}}$are isolated $\mathrm{Al}$ sites charge-balanced by a single $\mathrm{H}^{+}, \mathrm{Al}_{\text {pair }}^{\mathrm{H}^{+}, \mathrm{H}^{+}}$are paired $\mathrm{Al}$ sites chargebalanced by two $\mathrm{H}^{+}$sites, and $\mathrm{Al}_{\text {pair }}^{\mathrm{Co}^{2+}}$ are paired $\mathrm{Al}$ sites charge-balanced by a $\mathrm{Co}^{2+}$ ion. In this derivation, isolated $\mathrm{Co}^{2+}$ cations are assumed to be the only Co species present and can only exchanged at a paired $\mathrm{Al}$ site, while $\mathrm{H}^{+}$are the only monovalent cations present and can exchange at both isolated and paired Al sites. Substituting Eq. (S.2)-(S.4) into Eq. (S.1) results in a site balance equation relating $\mathrm{Al}_{\text {tot }}$ to the number of $\mathrm{Al}$ sites occupied by different cations: 


$$
A l_{\text {tot }}=A l_{\text {non,exch }}+A l_{\text {iso }}^{H^{+}}+2\left(A l_{\text {pair }}^{H^{+}, H^{+}}+A l_{\text {pair }}^{C O^{2+}}\right)
$$

The exchange of aqueous $\mathrm{Co}^{2+}$ ions $\left(\mathrm{Co}_{(a q)}^{2+}\right)$ onto paired $\mathrm{Al}$ sites occupied by two protons $\left(A l_{\text {pair }}^{H^{+} H^{+}}\right)$to form aqueous $\mathrm{H}^{+}\left(H_{(a q)}^{+}\right)$and paired $\mathrm{Al}$ sites occupied by $\mathrm{Co}^{2+}\left(\mathrm{Al}_{\text {pair }}^{\mathrm{Co}^{2+}}\right)$ is given by the following equilibrium reaction:

$$
C o_{(a q)}^{2+}+A l_{\text {pair }}^{H^{+} H^{+}} \stackrel{K}{\leftrightarrow} 2 H_{(a q)}^{+}+A l_{\text {pair }}^{C O^{2+}}
$$

where $\mathrm{K}$ is the equilibrium constant and is defined in terms of the following concentrations as:

$$
K=\frac{\left[H_{(a q)}^{+}\right]^{2}\left[A l_{\text {pair }}^{\mathrm{Co}^{2+}}\right]}{\left[\mathrm{Co}_{(\text {aq })}^{2+}\right]\left[A l_{\text {pair }}^{H^{+}, H^{+}}\right]}\left(\frac{1}{C^{0}}\right)
$$

where $\mathrm{C}^{0}$ is the standard state reference concentration of $1 \mathrm{M}$.

Dividing Eq. (S.5) by $\mathrm{Al}_{\text {tot }}$ yields a site balance equation expressed in terms of the fractional coverages $(\theta)$ for each species:

$$
1=\frac{A l_{\text {non,exch }}}{A l_{\text {tot }}}+\theta_{\text {iso }}^{H^{+}}+2\left(\theta_{\text {pair }}^{H^{+} H^{+}}+\theta_{\text {pair }}^{C O^{2+}}\right)
$$

where $\theta_{\text {iso }}^{H^{+}}$is the coverage of $A l_{\text {iso }}^{H^{+}}$species, $\theta_{\text {pair }}^{H^{+} H^{+}}$is the coverage of $A l_{\text {pair }}^{H^{+}, H^{+}}$species, and $\theta_{\text {pair }}^{\text {CO }^{2+}}$ is the coverage of $\mathrm{Al}_{\text {pair }}^{\mathrm{Co}^{2+}}$ and each coverage term is defined as follows:

$$
\begin{gathered}
\theta_{\text {iso }}^{H^{+}}=\frac{A l_{\text {iso }}^{H^{+}}}{A l_{\text {tot }}} \\
\theta_{\text {pair }}^{H^{+} H^{+}}=\frac{A l_{\text {pair }}^{H^{+} H^{+}}}{A l_{\text {tot }}} \\
\theta_{\text {pair }}^{C o^{2+}}=\frac{A l_{\text {pair }}^{C O^{2+}}}{A l_{\text {tot }}}
\end{gathered}
$$


Dividing Eq. (S.1) by $\mathrm{Al}_{\text {tot }}$ also results in an expression for the fraction of $\mathrm{Al}_{\text {exch }}\left(\frac{A l_{\text {exch }}}{A l_{\text {tot }}}\right)$ in terms of the fraction of $\mathrm{Al}_{\text {non.exch }}\left(\frac{A l_{\text {non,exch }}}{A l_{\text {tot }}}\right)$ :

$$
1-\frac{A l_{\text {non,exch }}}{A l_{\text {tot }}}=\frac{A l_{\text {exch }}}{A l_{\text {tot }}}
$$

Substituting Eq. (S.12) into Eq. (S.8) results in the following equation that describes the $\frac{A l_{\text {exch }}}{A l_{\text {tot }}}$ in terms of the fractional coverages of each species:

$$
\frac{A l_{\text {exch }}}{A l_{\text {tot }}}=\theta_{\text {iso }}^{H^{+}}+2\left(\theta_{\text {pair }}^{H^{+}, H^{+}}+\theta_{\text {pair }}^{C o^{2+}}\right)
$$

where $2\left(\theta_{\text {pair }}^{H^{+} H^{+}}+\theta_{\text {pair }}^{C o^{2+}}\right)$ is equal to the fraction of $\mathrm{Al}_{\text {pair }}$ present $\left(\frac{A l_{\text {pair }}}{A l_{\text {tot }}}\right)$

The equilibrium constant, K (Eq. (S.7)), can be rewritten in terms of coverages to derive an expression for $\theta_{\text {pair }}^{H^{+} H^{+}}$:

$$
\theta_{\text {pair }}^{H^{+}, H^{+}}=\frac{\left[H_{(a q)}^{+}\right]^{2} \theta_{\text {pair }}^{\text {Co }^{2+}}}{K\left[C o_{(a q)}^{2+}\right]}
$$

Eq. (S.14) can then be substituted into Eq. (S.13) to obtain an expression in terms of quantities that can be measured experimentally:

$$
\frac{A l_{\text {exch }}}{A l_{\text {tot }}}=\theta_{i s o}^{H^{+}}+2\left(\frac{\left[H_{(a q)}^{+}\right]^{2} \theta_{\text {pair }}^{C O^{2+}}}{K\left[\operatorname{Co}_{(a q)}^{2+}\right]}+\theta_{\text {pair }}^{C o^{2+}}\right)
$$

Eq. (S.15) can be rearranged to solve for $\theta_{\text {pair }}^{\mathrm{Co}^{2+}}$, which is a mathematical representation of the $\mathrm{Co}^{2+}$ exchange isotherm:

$$
\theta_{\text {pair }}^{C o^{2+}}=\left(\frac{1}{2}\right)\left(\frac{A l_{\text {exch }}}{A l_{\text {tot }}}-\theta_{\text {iso }}^{H^{+}}\right)\left(\frac{K\left[C o_{(a q)}^{2+}\right] /\left[H_{(a q)}^{+}\right]^{2}}{1+K\left[C o_{(a q)}^{2+}\right] /\left[H_{(a q)}^{+}\right]^{2}}\right)
$$


where the term $\left[\mathrm{Co}_{(a q)}^{2+}\right] /\left[H_{(a q)}^{+}\right]^{2}$ represents the ratio of $C o_{(a q)}^{2+}$ and $H_{(a q)}^{+}$in the exchange solution at equilibrium, and where the term $\left(\frac{1}{2}\right)\left(\frac{A l_{\text {exch }}}{A l_{\text {tot }}}-\theta_{\text {iso }}^{H^{+}}\right)$is equal to $\frac{A l_{\text {pair }}}{A l_{\text {tot }}}$ from Eq. (S.13) and represents the saturation limit of the $\mathrm{Co}^{2+}$ exchange isotherm.

$$
\theta_{\text {pair }}^{C o^{2+}}=\frac{A l_{\text {pair }}}{A l_{\text {tot }}}\left(\frac{K\left[C o_{(a q)}^{2+}\right] /\left[H_{(a q)}^{+}\right]^{2}}{1+K\left[C o_{(a q)}^{2+}\right] /\left[H_{(a q)}^{+}\right]^{2}}\right)
$$

Eq. (S.17) can be regressed to the experimental data to estimate $\mathrm{K}$ and $\frac{A l_{\text {pair }}}{A l_{\text {tot }}}$. The isotherms fit to the experimental data on both H-SSZ-13(15, 1) and H-SSZ-13(15, 0) are shown in Figure 1a of the main text. For H-SSZ-13(15, 1) the value of the equilibrium constant, K, was 0.027 and the value of $\frac{A l_{\text {pair }}}{A l_{\text {tot }}}$ was 0.087 , which is the predicted maximum number of sites capable of exchanging a divalent cation (e.g., $\left.\mathrm{Co}^{2+}\right)$. For H-SSZ-13(15,0), $\mathrm{K}$ was 0.016 and $\frac{A l_{\text {pair }}}{A l_{\text {tot }}}$ was 0.003 . 


\section{Section S.15. UV-Vis Spectroscopy of Co-SSZ-13(15, 1)}

Figure S.16. Ambient UV-Vis spectra of Co-SSZ-13(15, 1) with Co/Al values of 0.021 (faint grey trace), 0.022 (light grey trace), 0.047 (grey trace), 0.08 (dark grey trace), and 0.084 (black trace).

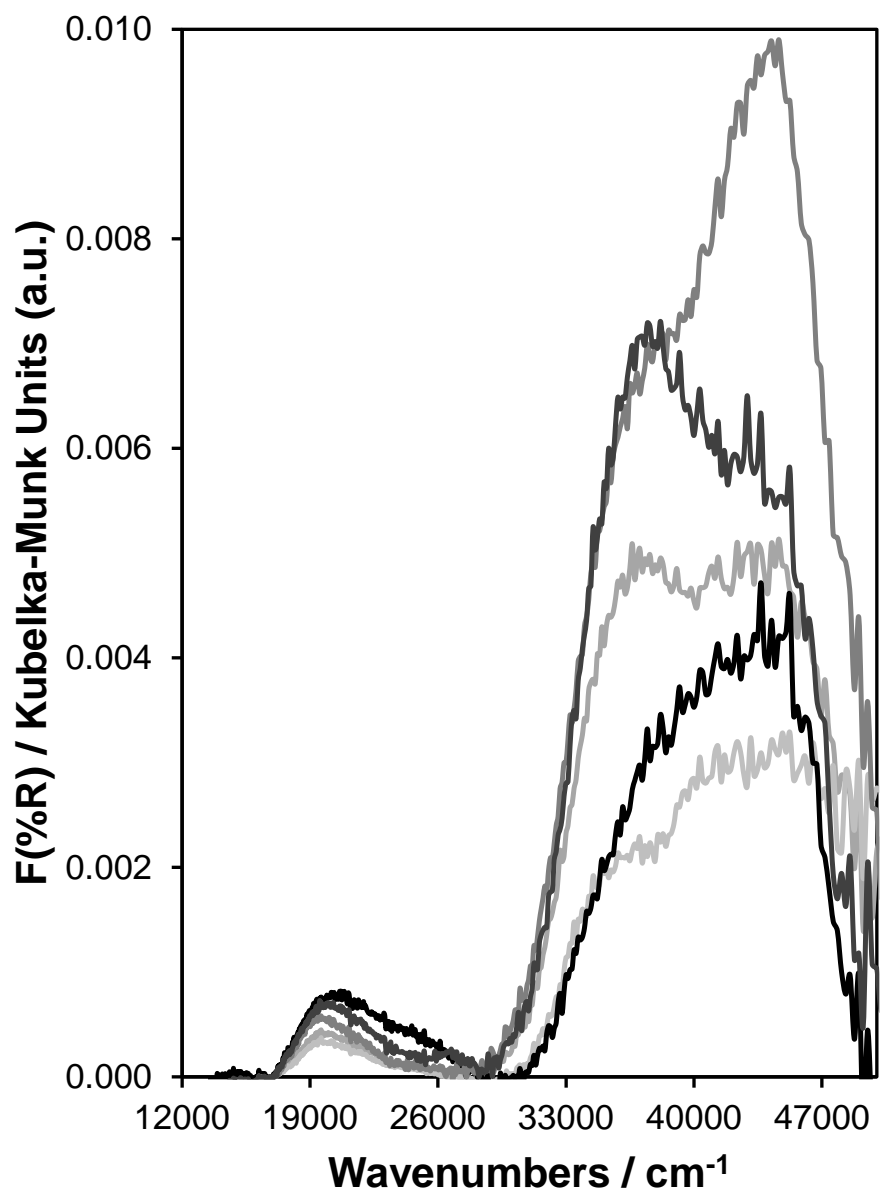




\section{Section S.16. Sodium Cation Exchange Procedure and Derivation of Ion-Exchange Isotherm}

A Langmuirian isotherm model for $\mathrm{Na}^{+}$exchange was derived starting from Eq. (S.1), but using only a single adsorption site to describe the $A l_{\text {exch }}$ since a monovalent cation cannot distinguish between isolated and paired $\mathrm{Al}$ sites. Therefore, $A l_{\text {exch }}$ can be described by the sum of the total number of $\mathrm{Al}$ exchange sites with a $\mathrm{H}^{+}$and with a $\mathrm{Na}^{+}$present:

$$
A l_{\text {exch }}=A l^{H^{+}}+A l^{N a^{+}}
$$

The equilibrium reaction of aqueous $\mathrm{Na}^{+}$ions $\left(\mathrm{Na}_{(a q)}^{+}\right)$with proton-occupied $\mathrm{Al}$ exchange sites $\left(A l^{H^{+}}\right)$to form aqueous $\mathrm{H}^{+}\left(H_{(a q)}^{+}\right)$and sodium-occupied $\mathrm{Al}$ sites $\left(A l^{N a^{+}}\right)$is given by:

$$
N a_{(a q)}^{+}+A l^{H^{+}} \stackrel{K}{\leftrightarrow} H_{(a q)}^{+}+A l^{N a^{+}}
$$

where $\mathrm{K}$ is the equilibrium constant defined in terms of concentrations as:

$$
K=\frac{\left[H_{(a q)}^{+}\right]\left[A l^{N a^{+}}\right]}{\left[N a_{(a q)}^{+}\right]\left[A l^{H^{+}}\right]}
$$

Substituting Eq. (S.18) into Eq. (S.1) and dividing by $\mathrm{Al}_{\text {tot }}$ provides an expression in terms of the fractional coverages $(\theta)$ of each species:

$$
1=\frac{A l_{\text {non,exch }}}{A l_{\text {tot }}}+\theta^{H^{+}}+\theta^{N a^{+}}
$$

where $\theta^{H^{+}}$is the coverage of $\mathrm{Al}^{\mathrm{H}^{+}}$and $\theta^{N a^{+}}$is the coverage of $\mathrm{Al}^{N a^{+}}$defined as follows:

$$
\begin{gathered}
\theta^{H^{+}}=\frac{A l^{H^{+}}}{A l_{t o t}} \\
\theta^{N a^{+}}=\frac{A l^{N a^{+}}}{A l_{t o t}}
\end{gathered}
$$

Substituting Eq. (S.12) into Eq. (S.21) results in an equation that describes $\frac{A l_{\text {exch }}}{A l_{\text {tot }}}$ in terms of the fractional coverage of $\mathrm{H}^{+}$and $\mathrm{Na}^{+}$: 


$$
\frac{A l_{\text {exch }}}{A l_{t o t}}=\theta^{H^{+}}+\theta^{N a^{+}}
$$

The equilibrium constant, K (Eq. (S.20)), can be rewritten in terms of coverages to obtain an explicit expression for $\theta^{H^{+}}$:

$$
\theta^{H^{+}}=\frac{\left[H_{(a q)}^{+}\right] \theta^{N a^{+}}}{K\left[N a_{(a q)}^{+}\right]}
$$

Eq. (S.25) can be substituted into Eq. (S.24) to obtain an expression in terms of quantities that can be measured experimentally:

$$
\frac{A l_{\text {exch }}}{A l_{\text {tot }}}=\theta^{N a^{+}}\left(1+\frac{\left[H_{(a q)}^{+}\right]}{K\left[N a_{(a q)}^{+}\right]}\right)
$$

Eq. (S.26) can be rearranged to obtain an expression for $\theta^{\mathrm{Na}^{+}}$, which is the $\mathrm{Na}^{+}$exchange isotherm:

$$
\theta^{N a^{+}}=\left(\frac{A l_{\text {exch }}}{A l_{\text {tot }}}\right)\left(\frac{K\left[N a_{(a q)}^{+}\right] /\left[H_{(a q)}^{+}\right]}{1+K\left[N a_{(a q)}^{+}\right] /\left[H_{(a q)}^{+}\right]}\right)
$$

The term $\left[N a_{(a q)}^{+}\right] /\left[H_{(a q)}^{+}\right]$represents the ratio of $N a_{(a q)}^{+}$and $H_{(a q)}^{+}$ions in the exchange solution at equilibrium. Eq. (S.27) can be regressed to the experimental data to estimate $\mathrm{K}$ and $\frac{A l_{\text {exch }}}{A l_{\text {tot }}}$. The isotherm fit to the experimental data is shown in Figure $1 \mathrm{~b}$ of the main text. For H-SSZ-13(15, 0) the value of the equilibrium constant, $\mathrm{K}$, was 0.055 and the value of $\frac{A l_{\text {exch }}}{A l_{\text {tot }}}$ was 1.02 . For H-SSZ$13(15,1), \mathrm{K}$ was 0.14 and the value of $\frac{A l_{\text {exch }}}{A l_{\text {tot }}}$ was 0.91 , which is in agreement with the experimental results for the $\mathrm{H}^{+} / \mathrm{Al}$ determined from $\mathrm{NH}_{3}$ TPD (Table 2 in main text). 
Section S.17. Characterization Data for SSZ-13(15) with Varying $\left(\mathrm{Na}^{+}+\mathrm{TMAda}^{+}\right) / \mathrm{Al}(\mathrm{Total}$ Charge) and Varying $\mathrm{Na}^{+} / \mathrm{TMAda}^{+}$Ratios (Charge Density)

Table S.3. Characterization for SSZ-13 samples synthesized at a constant $\mathrm{Na}^{+} / \mathrm{TMAda}^{+}=0.67$ and varying $\mathrm{Si} / \mathrm{Al}$.

\begin{tabular}{cccccccc}
\hline Sample & Si/Al & $\begin{array}{c}\text { Micropore } \\
\text { Volume } \\
\left(\mathbf{c m}^{\mathbf{3}} \mathbf{g}^{-1}\right)\end{array}$ & $\mathbf{H}^{+} / \mathbf{A l}$ & $\mathbf{N a}^{+} / \mathbf{A l}$ & $\mathbf{T M A d a}{ }^{+} / \mathbf{A l}$ & $\mathbf{( N a}^{+}+\mathbf{T M A d a} \mathbf{H}^{+} / \mathbf{A l}$ & $\mathbf{C o} / \mathbf{A l}$ \\
\hline SSZ-13(10, 0.67) & 10.0 & - & 0.83 & 0.11 & - & - & 0.07 \\
SSZ-13(15, 0.67) & 15.0 & 0.16 & 0.98 & 0.06 & 0.93 & 0.99 & 0.06 \\
SSZ-13(25, 0.67) & 21.1 & - & - & 0.08 & - & - & 0.03 \\
SSZ-13(50, 0.67) & 36.2 & - & - & 0.01 & - & - & 0.01 \\
SSZ-13(100, 0.67) & 65.0 & - & - & 0.04 & - & - & 0.01 \\
\hline
\end{tabular}


Section S.18. Characterization Data for SSZ-13(15) with Varying $\left(\mathrm{Na}^{+}+\mathrm{TMAda}{ }^{+}\right) / \mathrm{Al}$ (Total Charge) and Varying $\mathrm{Na}^{+} / \mathrm{TMAda}^{+}$Ratios (Charge Density)

Table S.4. Characterization data for SSZ-13(15) and MOR samples synthesized with constant synthesis solution $\mathrm{Si} / \mathrm{Al}$ and varying total solution charge and $\mathrm{Na}^{+} / \mathrm{TMAda}^{+}$.

\begin{tabular}{|c|c|c|c|c|c|c|c|}
\hline Sample & Si/Al & $\begin{array}{c}\text { Micropore } \\
\text { Volume } \\
\left(\mathrm{cm}^{3} \mathbf{g}^{-1}\right) \\
\end{array}$ & $\mathbf{H}^{+} / \mathbf{A l}$ & $\mathrm{Na}^{+} / \mathrm{Al}$ & TMAda $^{+} / \mathbf{A l}$ & $\left(\mathrm{Na}^{+}+\mathrm{TMAda}^{+}\right) / \mathrm{Al}$ & $\mathrm{Co} / \mathrm{Al}$ \\
\hline SSZ-13(15,0) & 17.5 & 0.20 & 0.91 & 0.01 & 1.20 & 1.21 & 0.00 \\
\hline SSZ-13(15, 0.35) & 17.0 & - & - & 0.01 & 1.06 & 1.07 & 0.03 \\
\hline SSZ-13(15, 0.70) & 15.5 & 0.18 & - & 0.04 & 0.89 & 0.93 & 0.04 \\
\hline SSZ-13(15, 0.93) & 15.1 & 0.16 & - & 0.05 & 0.94 & 0.99 & 0.06 \\
\hline SSZ-13(15, 1.38) & 13.3 & - & 0.96 & 0.04 & 0.92 & 0.96 & 0.08 \\
\hline SSZ-13(15, 2.09) & 11.6 & - & - & 0.02 & 1.01 & 1.03 & 0.06 \\
\hline $\operatorname{MOR}(15,3.45)$ & 11.9 & - & - & 0.08 & - & - & 0.03 \\
\hline $\operatorname{MOR}(15,4.16)$ & 11.9 & - & - & 0.09 & - & - & 0.01 \\
\hline $\operatorname{MOR}(15,5.55)$ & 9.9 & - & 0.94 & 0.10 & - & - & 0.02 \\
\hline
\end{tabular}

Figure S.17. Si/Al ratio as a function of $\mathrm{Na}^{+} / \mathrm{TMAda}^{+}$ratio for SSZ-13(15) samples synthesized with (squares) and without (circles) a constant $\left(\mathrm{Na}^{+}+\mathrm{TMAda}^{+}\right) / \mathrm{Al}$ ratio. The dashed line is a linear fit to the samples synthesized without constant $\left(\mathrm{Na}^{+}+\mathrm{TMAda}^{+}\right) / \mathrm{Al}$ (circles).

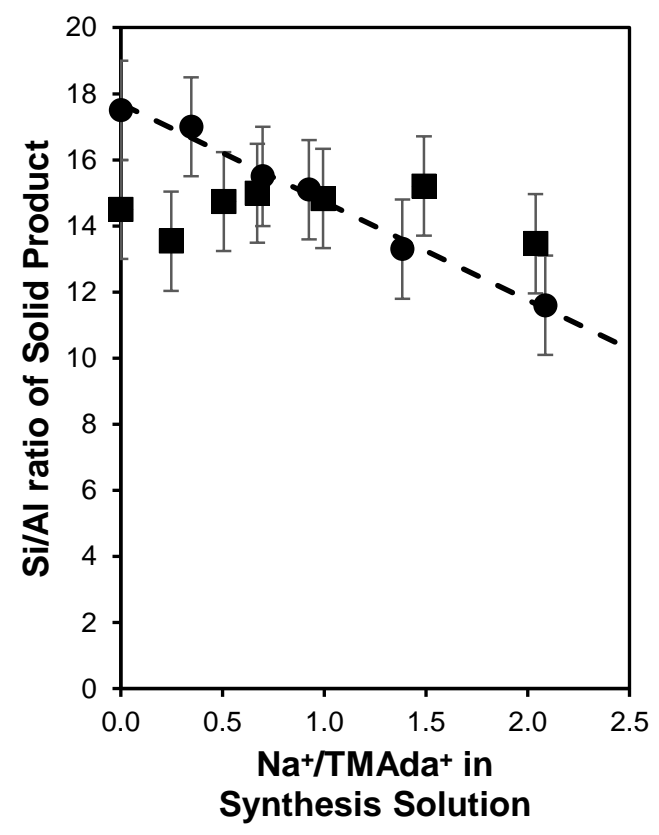


Section S.19. Characterization Data for SSZ-13(25) with Constant $\left(\mathrm{Na}^{+}+\mathrm{TMAda}{ }^{+}\right) / \mathrm{Al}(\mathrm{Total}$ Charge) and Varying $\mathrm{Na}^{+} / \mathrm{TMAda}^{+}$Ratios (Charge Density)

Table S.5. Characterization data for SSZ-13(25) samples synthesized with constant synthesis solution $\mathrm{Si} / \mathrm{Al}$ and total solution charge and varying $\mathrm{Na}^{+} / \mathrm{TMAda}^{+}$.

\begin{tabular}{cccccccc}
\hline Sample & Si/Al & $\begin{array}{c}\text { Micropore } \\
\text { Volume } \\
\left(\mathbf{c m}^{3} \mathbf{g}^{-1}\right)\end{array}$ & $\mathbf{H}^{+} / \mathbf{A l}$ & $\mathbf{N a}^{+} / \mathbf{A l}$ & $\mathbf{T M A d a}{ }^{+} / \mathbf{A l}$ & $\left(\mathbf{N a}^{+}+\mathbf{T M A d a}^{+}\right) / \mathbf{A l}$ & $\mathbf{C o} / \mathbf{A l}$ \\
\hline SSZ-13(25, 0.25) & 25.3 & 0.19 & - & 0.05 & 1.04 & 1.09 & 0.03 \\
SSZ-13(25, 0.50) & 24.7 & 0.19 & - & 0.11 & 0.96 & 1.07 & 0.03 \\
SSZ-13(25, 1.00) & 24.1 & 0.20 & 1.02 & 0.06 & 1.01 & 1.07 & 0.04 \\
SSZ-13(25, 2.00) & 26.1 & 0.20 & - & 0.05 & 0.94 & 0.99 & 0.02 \\
\hline
\end{tabular}

Figure S.18. Fraction of paired $\mathrm{Al}$ atoms measured by titration with $\mathrm{Co}^{2+}$ as a function of the synthesis solution charge density on SSZ-13(25) synthesized with constant total solution charge.

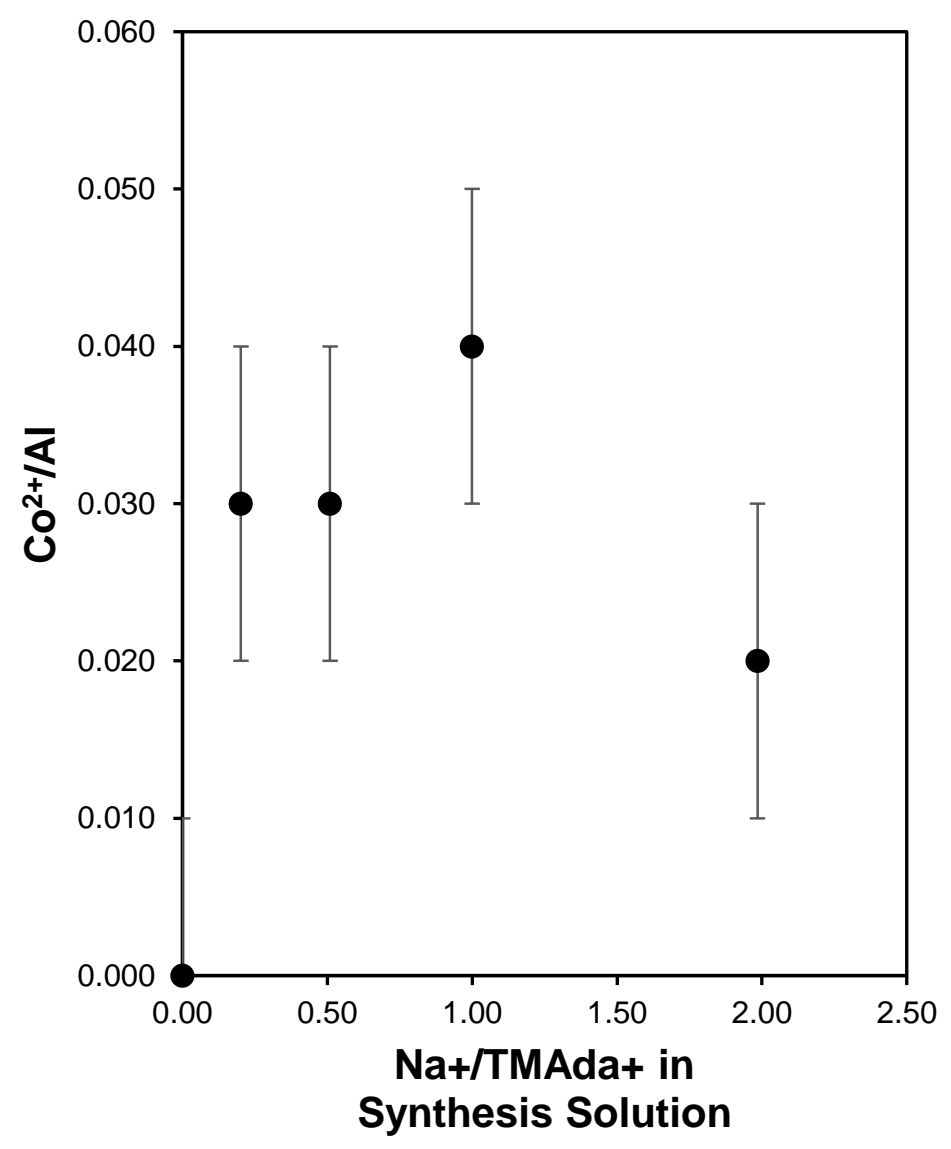




\section{REFERENCES}

(1) Fickel, D. W.; Fedeyko, J. M.; Lobo, R. F. Copper Coordination in Cu-SSZ-13 and Cu-SSZ16 Investigated by Variable-Temperature XRD, J. Phys. Chem. C 2010, 114, 1633-1640.

(2) Zones, S. I. Conversion of Faujasites to High-Silica Chabazite SSZ-13 in the Presence of N,N,N-trimethyl-1-adamantammonium Iodide, J. Chem. Soc., Faraday Trans. 1991, 87, 37093716.

(3) Deka, U.; Juhin, A.; Eilertsen, E. A.; Emerich, H.; Green, M. A.; Korhonen, S. T.;

Weckhuysen, B. M.; Beale, A. M. Confirmation of Isolated Cu2+ Ions in SSZ-13 Zeolite as Active Sites in NH3-Selective Catalytic Reduction, J. Phys. Chem. C 2012, 116, 4809-4818.

(4) Zones, S. I. US4544538 A, October 1, 1985

(5) Díaz-Cabañas, M. J.; Barrett, P. A.; Camblor, M. A. Synthesis and Structure of Pure SiO2 Chabazite: The SiO2 Polymorph with the Lowest Framework Density, Chem. Commun. 1998, 1881-1882.

(6) Gounder, R.; Jones, A. J.; Carr, R. T.; Iglesia, E. Solvation and Acid Strength Effects on Catalysis by Faujasite Zeolites, J. Catal. 2012, 286, 214-223.

(7) Kraus, H.; Müller, M.; Prins, R.; Kentgens, A. P. M. Comments on the 27Al NMR Visibility of Aluminas, J. Phys. Chem. B 1998, 102, 3862-3865.

(8) Luz, Z.; Vega, A. J. Interaction of H-RHO Zeolite with Water and Methanol Studied by Multinuclear NMR Spectroscopy, J. Phys. Chem. 1987, 91, 374-382.

(9) Bates, S. A.; Verma, A. A.; Paolucci, C.; Parekh, A. A.; Anggara, T.; Yezerets, A.;

Schneider, W. F.; Miller, J. T.; Delgass, W. N.; Ribeiro, F. H. Identification of the Active Cu Site in Standard Selective Catalytic Reduction with Ammonia on Cu-SSZ-13, J. Catal. 2014, 312, 87-97. 